ARTICLE

Received 11 Nov 2015 | Accepted 26 Feb 2016 | Published 11 Apr 2016

DOI: $10.1038 /$ ncomms11191

OPEN

\title{
Unconventional endosome-like compartment and retromer complex in Toxoplasma gondii govern parasite integrity and host infection
}

Lamba Omar Sangaré1, Tchilabalo Dilezitoko Alayi ${ }^{2,3}$, Benoit Westermann², Agnes Hovasse², Fabien Sindikubwabo ${ }^{4}$, Isabelle Callebaut ${ }^{5}$, Elisabeth Werkmeister ${ }^{6}$, Frank Lafont ${ }^{6}$, Christian Slomianny ${ }^{7}$, Mohamed-Ali Hakimi ${ }^{4}$, Alain Van Dorsselaer ${ }^{2}$, Christine Schaeffer-Reiss ${ }^{2}$ \& Stanislas Tomavo,3

Membrane trafficking pathways play critical roles in Apicomplexa, a phylum of protozoan parasites that cause life-threatening diseases worldwide. Here we report the first retromer-trafficking interactome in Toxoplasma gondii. This retromer complex includes a trimer Vps35-Vps26-Vps29 core complex that serves as a hub for the endosome-like compartment and parasite-specific proteins. Conditional ablation of $\mathrm{Tg} \vee p s 35$ reveals that the retromer complex is crucial for the biogenesis of secretory organelles and for maintaining parasite morphology. We identify $\mathrm{TgHP} 12$ as a parasite-specific and retromer-associated protein with functions unrelated to secretory organelle formation. Furthermore, the major facilitator superfamily homologue named TgHPO3, which is a multiple spanning and ligand transmembrane transporter, is maintained at the parasite membrane by retromer-mediated endocytic recycling. Thus, our findings highlight that both evolutionarily conserved and unconventional proteins act in concert in T. gondii by controlling retrograde transport that is essential for parasite integrity and host infection.

\footnotetext{
${ }^{1}$ Center for Infection and Immunity of Lille, INSERM U 1019, CNRS UMR 8204, Institut Pasteur de Lille, Université de Lille, 59000 Lille, France. ${ }^{2}$ Laboratory of Bio-Organic Mass Spectrometry, IPHC, CNRS UMR 7178, Université de Strasbourg, 67087 Strasbourg, France. ${ }^{3}$ Plateforme de Protéomique et des Peptides Modifiés (P3M), Institut Pasteur de Lille, CNRS, Université de Lille, 59000 Lille, France. ${ }^{4}$ CNRS UMR5163, LAPM, Université Joseph Fourier, Grenoble 38000 , France. ${ }^{5}$ CNRS UMR7590, Sorbonne Universités, Université Pierre et Marie Curie-Paris 6, MNHN, IRD-IUC, Paris 75005, France. ${ }^{6}$ Bioimaging Platform, IBL, CNRS, Université de Lille, 59000 Lille, France. ${ }^{7}$ Laboratory of Cell Physiology, INSERM U 1003, Université de Lille, 59655 Villeneuve d'Asca, France. Correspondence and requests for materials should be addressed to S.T. (email: stanislas.tomavo@gmail.com).
} 
T he phylum Apicomplexa comprises an ancient group of early divergent eukaryotes, including some of the most deadly pathogens of medical and veterinary importance. Plasmodium species are responsible for malaria, which causes as many as 700,000 deaths per year, while Toxoplasma gondii chronically infects up to $30 \%$ of the human population, with immunocompromised patients and pregnant women at risk for adverse outcomes, such as toxoplasmic encephalitis and spontaneous abortion, respectively ${ }^{1}$. T. gondii is considered a model system not only for its pathogenic relatives but also for intracellular parasitism and infection biology in general. T. gondii has common eukaryotic organelles, including the nucleus, endoplasmic reticulum and a single Golgi stack, but also specific secretory organelles named dense granules, micronemes and rhoptries that contain parasite-derived factors required for host infection. Rhoptries and micronemes are formed de novo during parasite replication, and this process requires significant protein and lipid trafficking through the secretory pathway.

The trafficking mechanisms employed by $T$. gondii retain several typical eukaryote components as well as evolving divergent features. Protein trafficking of this parasite is mediated by entry into a canonical endoplasmic reticulum followed by vesicle packaging through a single Golgi complex ${ }^{2,3}$. Post-Golgi protein sorting to specific organelles requires the function of dynamin-related protein $\mathrm{B}$, which is involved in fission events ${ }^{4}$. Downstream Rab-GTPases function throughout the parasite secretory pathway ${ }^{5}$. T. gondii soluble $N$-ethylmaleimidesensitive-factor attachment protein receptor (SNARE) proteins in docking and fusion at target membranes have also been described ${ }^{6,7}$. However, unlike in mammalian cells, T. gondii endoplasmic reticulum is reduced so that the nuclear envelope itself contributes to a substantial proportion of its total volume ${ }^{2}$. Whereas in mammalian cells hundreds of Golgi stacks occupy the perinuclear $\operatorname{area}^{8}$, the Golgi apparatus is limited to a single discrete structure in T. gondii. The post-Golgi system, also named the endosome-like compartment (ELC), is involved in the trafficking of microneme proteins ${ }^{10,11}$. The ELC is decorated by the small GTPases, Rab5 and Rab7, which are typically associated with the endosomal system. Nevertheless, classical endocytosis has not yet been validated in T. gondii. This parasite has no lysosomes; rather the parasite harbours acidic vesicles that were thought to be precursors of the rhoptry organelles ${ }^{12}$. The parasite lacks most components of endosomal sorting complexes, which are known for their roles in forming multivesicular bodies that deliver ubiquitinated membrane proteins and lipids to lysosomes for degradation ${ }^{3,13}$. The machinery required for caveogenesis and caveola-dependent invaginations have not yet been identified in the parasite ${ }^{14}$. Furthermore, while evidence of conventional clathrin-dependent endocytosis by $T$. gondii is lacking, clathrin is present exclusively in post-Golgi compartments where its function is restricted to post-Golgi trafficking ${ }^{15}$, and the uptake of cytosol proteins by the tachyzoites of $T$. gondii has recently been described using an endocytosis assay ${ }^{16}$. However, the mechanisms underlying the events of this unconventional endocytosis in the parasite remain to be determined. Clearly, the secretory pathway of $T$. gondii can be considered a strippeddown version of the more complex trafficking machinery that characterizes higher eukaryotes. Despite this minimal trafficking machinery, the parasites actively rely on a membrane vesicle formation and transport during its intracellular lifecycle; however, to date, comparatively little is known about the mechanisms involved in trafficking pathways in T. gondii.

We previously reported a $T$. gondii sortilin-like receptor (TgSORTLR) that regulates protein transport and is essential for apical secretory organelle biogenesis and host infection ${ }^{17}$.
Moreover, the C-terminal tail of TgSORTLR was shown to be involved in recruiting many cytosolic cargo proteins including two homologues of the core retromer components, Vps26 and Vps35 (ref. 17), which are known to regulate retrograde transport from endosomes to the trans-Golgi network (TGN) in yeast and mammals $^{18,19}$.

Here, we report that a singular architecture with a trimer Vps35-Vps26-Vps29 core complex acts as the major endosomal cargo recycling machinery and is required for parasite integrity and more specifically for secretory organelle biogenesis and maintenance of a multiple ligand-binding transporter at the T. gondii membrane. Our findings provide strong evidence that the unconventional TgSORTLR-containing ELC is involved in distinct mechanisms for the delivery of major retromerdependent cargo. They also demonstrate a role for the endocytic recycling pathway in $T$. gondii pathogenesis.

\section{Results}

Features of the retromer interactome of $T$. gondii. To identify proteins that interact with the $T$. gondii retromer complex, we chromosomally appended an encoded hemagglutinin (HA) epitope to $T g \mathrm{Vsp} 35$ and $T g \mathrm{Vps} 26$. This knock-in strategy allows steady-state levels of epitope-tagged protein expression via homologous promoters. We also tagged $T g \mathrm{Vps} 29$ identified in the parasite genome (TGME49_252490, www.toxodb.org) with a cMyc epitope as above. We performed a series of immunoprecipitation experiments under native conditions; revealing that $T g \mathrm{Vps} 35-\mathrm{HA}, \mathrm{TgVps} 26-\mathrm{HA}$ and $T g \mathrm{Vps} 29-\mathrm{cMyc}$ were specifically pulled down (Fig. 1a, lanes 2-4; and Supplementary Fig. 1) using HA or cMyc-tagged protein extracts and antibodies specific to $\mathrm{HA}$ and cMyc, respectively. No protein signals were detected in the negative controls using naive sera and the same protein extracts, as expected (Fig. 1a, lanes 5 and 6). In addition, immunoprecipitation of $T g \mathrm{Vps} 35-\mathrm{HA}$ and $\mathrm{TgVps} 26-\mathrm{HA}$ also revealed a faint protein band corresponding to TgSORTLR protein using rat antibodies anti-TgSORTLR (Fig. 1b, lane 3 (E) and blue stars in left and middle panels) while immunoprecipitation of $T g V p s 29$ did not, most likely due to its low-expression level (Fig. 1b, lane 3 (E), right panel). Mass spectrometry analysis of the eluates corroborates the presence of $T g \mathrm{Vps} 35, T g \mathrm{Vps} 26$ and $\mathrm{TgVps} 29$ in each immunoprecipitation sample (Supplementary Data 1). Consistent with the immunoblots shown in Fig. 1b, the presence of TgSORTLR was only confirmed in immunoprecipitates of $T g \mathrm{Vps} 35$ and $\mathrm{TgVps} 26$ by mass spectrometry (Supplementary Data 1). To gain unbiased insight into the genuine retromer composition in T. gondii, we developed a quantitative approach using micro liquid chromatography-selected reaction monitoring (microLC-SRM) and stable isotope-labelled standard peptides. The absolute quantification of $\mathrm{TgVps} 35$, $T g V p s 26$ and $T g V p s 29$ was carried out using three proteotypic peptides per protein (Supplementary Data 2). This approach yielded a stoichiometry of $\sim 1: 1$ for $T g V p s 35$ relative to $T g V p s 26$ and 3:1 between $T g V$ sp35 and $T g V p s 29$ (Supplementary Table 1). This stoichiometry between $T g V p s 35$ and TgVPS29 is in contrast to the formation of a functional core retromer complex at a ratio of $1: 1: 1$, as in mammalian and yeast cells ${ }^{20}$. However, this discrepancy may also be explained by the fact that $T g V P S 29$ may associate with $\operatorname{TgVPS} 35$ at a much lower affinity than $T g \mathrm{VPS} 26$, thus leading to the reduced levels of TgVPS29 identified by co-immunoprecipitations. In addition, 17 retromer-interacting proteins were identified in the interactome (Fig. 1c) and ranked according to the following filtering criteria: protein common to at least two co-immunoprecipitations, absent in the control and identified with at least two unique peptides (Supplementary Data 1). Most interactors (12 out of 17) were immunoprecipitated 


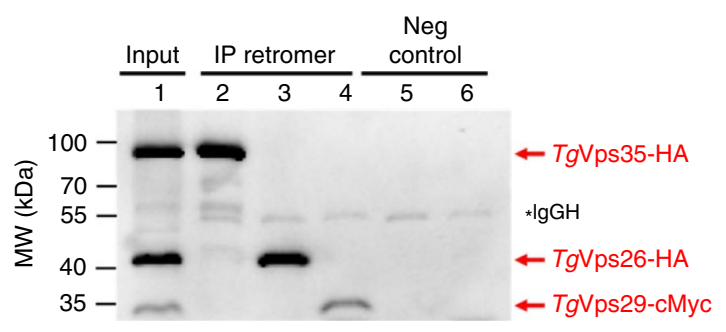

C

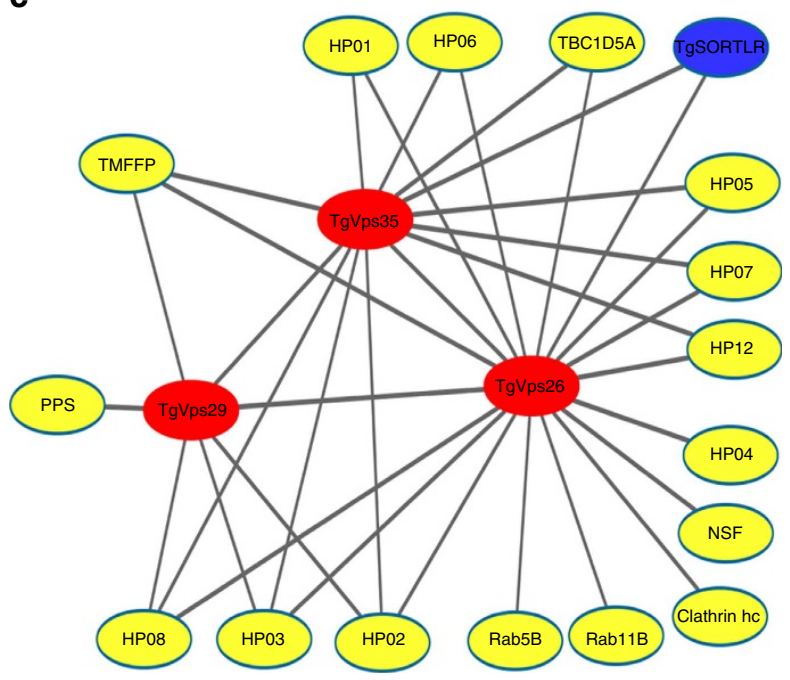

b

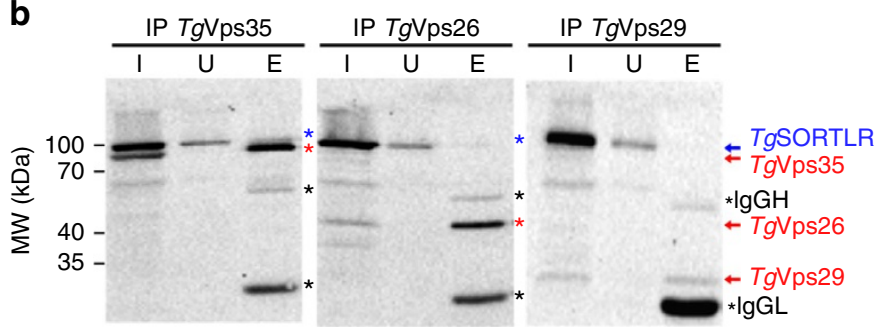

d
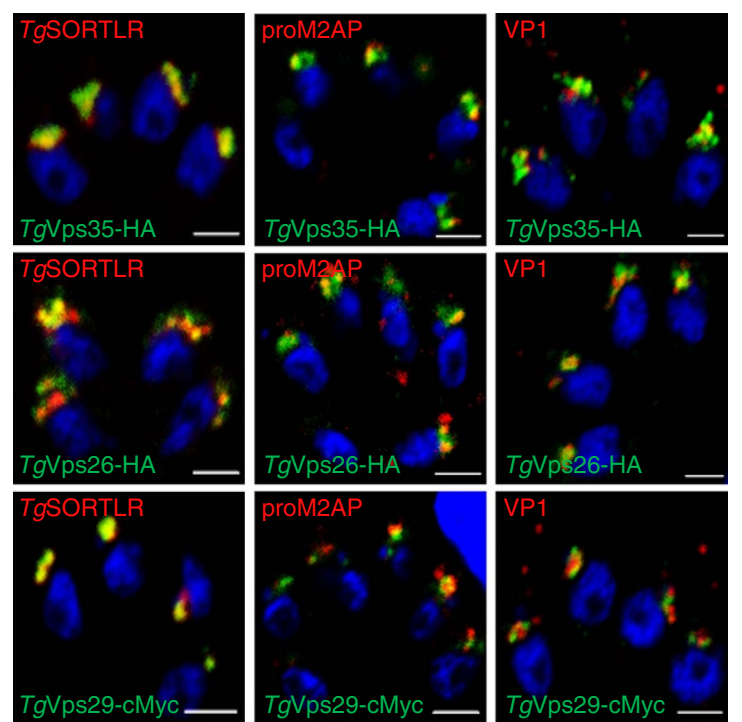

Figure 1 | Interactome reveals cargo-selective complex and other interactors with the retromer of $\boldsymbol{T}$. gondii. (a) Immunoblots of co-immunoprecipitates of $\mathrm{Tg} \mathrm{Vps} 35-\mathrm{HA}$ and $\mathrm{Tg} \mathrm{Vps} 26-\mathrm{HA}$ (designated IP retromer) probed with rabbit polyclonal specific anti-HA antibodies (lanes 2 and 3 ) using total detergent protein extracts from the knopck-in $\mathrm{TgVps} 35-\mathrm{HA}$ and $\mathrm{TgVs} 26-\mathrm{HA}$ parasites, respectively, and anti-HA beads. Immunoblot of co-immunoprecipitate of $\mathrm{Tg} \vee p s 29-c M y c$ (IP retromer) probed with rabbit polyclonal anti-cMyc antibodies (lane 4) using total detergent protein extract from the knock-in TgVps29cMyc parasites and anti-cMyc beads. Negative controls (Neg control) using total detergent protein extracts from untagged parental RH TaTi parasites incubated with anti-HA (lane 5) and anti-cMyc (lane 6) beads. Lane 1 (designated input) corresponds to equally mixed sample of all three detergent extracts containing $\mathrm{Tg} V p s 35-\mathrm{HA}, \mathrm{TgVps} 26-\mathrm{HA}$ and $\mathrm{Tg} \mathrm{Vps} 29-\mathrm{cMyc}$ proteins and revealed by a mixed probe containing both anti-HA and anti-cMyc antibodies. Molecular weights ( $\mathrm{kDa}$ ) of protein markers are shown on left. IgG $\mathrm{h}_{\mathrm{h}}$ means heavy chain of $\mathrm{IgG}$. (b) Immunoblots of $\mathrm{TgVps} 35-\mathrm{HA}$, $\mathrm{Tg} \vee \mathrm{ps} 26-\mathrm{HA}$ and $\mathrm{TgVsp29-cMyc}$ as described in a probed with rat specific anti-TgSORTLR antibodies. (I, input) corresponds to total detergent protein extracts from TgVps35-HA, TgVps26-HA and TgVps29-cMyc knock-in parasites, respectively; (U) unbound lysates to the anti-HA or anti-cMyc beads and (E) eluates corresponding to co-immunoprecipitates. The blots were simultaneously incubated with rat anti-TgSORTLR and rabbit anti-HA or rat anti-TgSORTLR and

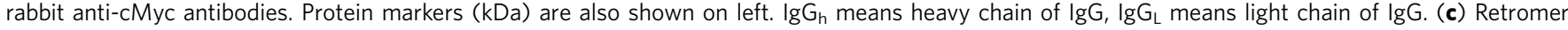
interactome was constructed by analysing the co-immunoprecipitates of $\mathrm{Tg} \mathrm{Vsp} 35-\mathrm{HA}, \mathrm{TgVps} 26-\mathrm{HA}$ and $\mathrm{TgVps} 29-\mathrm{cMyc}$ validated by immunoblotting in a and mass spectrometry (Supplementary Data 1). The interactome identified TgVps35, TgVps29 and TgVps26 (red) and TgSORTLR (blue) in addition to the putative phosphatidylinositol synthase (PPS), transporter major facilitator family protein (TMFFP), putative N-ethylmaleimide sensitive fusion protein (NSF), multi-pass transmembrane protein (MTP), Rab5, Rab11B, Rab7-GTPase-activating protein (GAP) regulator TBC1D5A homologue and nine parasitespecific HP. (d) Confocal imaging of TgVps35, TgVps26 and TgVps29 that co-localize with TgSORTLR, proM2AP and vacuolar protein 1 (VP1) using intracellular tachyzoites of the respective knock-in parasites stained with anti-HA or anti-cMyc antibodies followed by probing with anti-TgSORTLR, antiproM2AP and anti-VP1 antibodies, respectively. Bar, $2 \mu \mathrm{m}$.

with both $T g \mathrm{Vps} 35$ and $T g \mathrm{Vps} 26$ (Fig. 1c), confirming the potential of predominant $\mathrm{TgVps} 35-\mathrm{TgVsp} 26$ complexes in which only a fraction of $\mathrm{TgVsp} 29$ is bound to generate a functional retromer complex, as determined by the quantitative proteomics described in Supplementary Table 1. Functional classification by gene ontology analysis revealed that some of these interactors played roles in cell trafficking: Rab5B, an endosome marker; Rab11B, a factor essential for inner membrane complex recycling ${ }^{21}$; the TBC1D5A homologue, a Rab7-GTPaseactivating protein that negatively regulates the core retromer function $^{22}$; the aforementioned TgSORTLR receptor ${ }^{17}$; and $\mathrm{N}$-ethylmaleimide-sensitive protein, a factor involved in SNARE-dependent membrane fusion. In addition to the established binding partners, 9 out of 17 proteins are new parasite-specific proteins (that is, hypothetical proteins (HP);
Fig. 1c). Confocal imaging revealed that $\mathrm{TgVps} 35-\mathrm{HA}$, $T g V p s 26-H A$ and $T g V p s 29-c M y c$ co-localize with the ELC markers pro-microneme 2-associated protein (proM2AP), vacuolar protein 1 (VP1) and TgSORTLR (Fig. 1d). We therefore conclude that the ELC defines the sub-cellular compartment where retromer-mediated vesicle recycling or retrograde trafficking operates via an endolysosomal-like system in T. gondii.

$T g$ Vps35 silencing abrogates host infection by $T$. gondii. To establish the functional roles of $\mathrm{TgVps} 35$ in $T$. gondii infection, we generated conditional anhydrotetracyclin (ATc)-inducible knockout mutants (iKoTgVps35) using the strategy described in Fig. 2a. We selected three positive clones from the emerging stable parasite population and the genome editing of these clones 
a

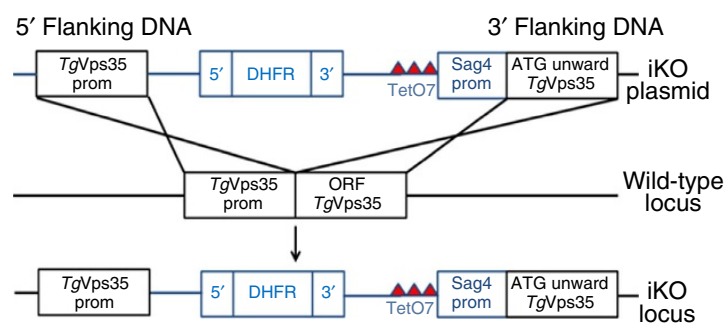

C

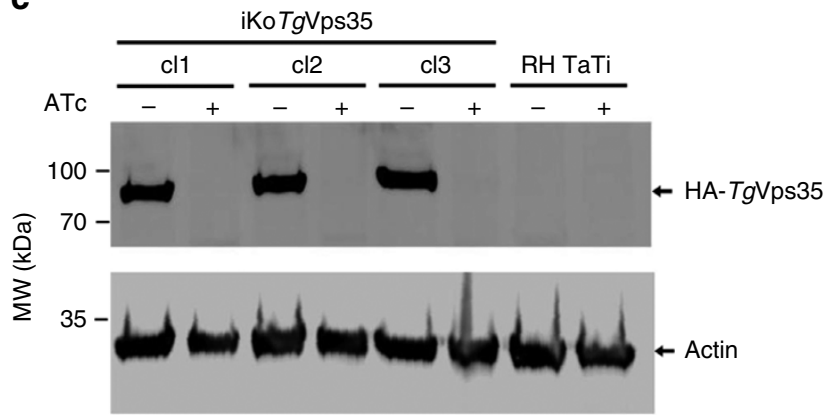

b

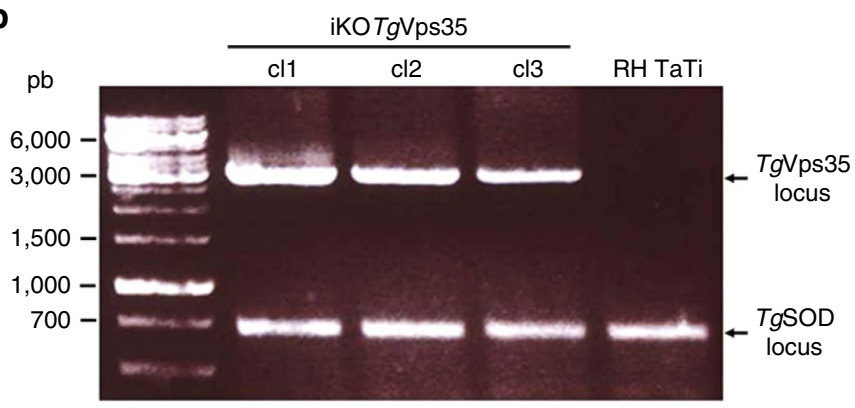

d

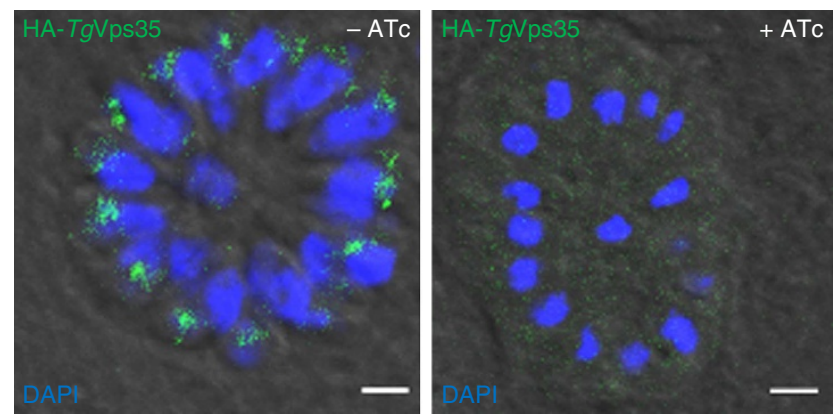

Figure 2 | Conditional ablation of $\mathbf{T g V p s} 35$ gene. (a) Schematic of the vector and experimental approach used for the conditional ablation of the TgVps35 gene. (b) PCR analysis of three clones with conditional disruption of $T g \vee p s 35$ and the parental line. Superoxide dismutase (SOD) served as the positive control. Also see the primers used for these PCR in Supplementary Table 3. (c) Immunoblots of the three conditional iKoTgVps35 mutants and RH TaTi parasites, which were grown in the presence or absence of ATc for $48 \mathrm{~h}$, harvested and purified. Each lane refers to a total SDS-protein extract corresponding to the equivalent of $2 \times 10^{6}$ parasites. Immunoblots were probed with anti-HA antibodies. Actin probed with specific monoclonal antibodies served as a loading control. Molecular weights $(\mathrm{kDa})$ of protein markers are indicated on left. (d) Intracellular vacuole containing 16-daughter iKoTgVps35 mutants corresponding to one of the three clones analysed by PCR and western blots and PCR confirmed the conditional depletion TgVps35 protein (right panel) by confocal imaging after $48 \mathrm{~h}$ post-infection in the presence of ATc. The left panel showed the same mutant in which $T g V p s 35$ protein was detected in the endosome-like comportment (ELC) closely located to the nuclei in the absence of ATc, as expected. 4', 6-diamidino-2-phenylindole (DAPI) was used to stain nuclei. Rabbit specific anti-HA antibodies was also used. Bar, $2 \mu \mathrm{m}$.

was verified by PCR, demonstrating the perfect integration of the knockout vector at the TgVps35 locus (Fig. 2b). Following ATc treatment, we subsequently observed the disappearance of HA- $T g V p s 35$ protein by western blotting (Fig. 2c) and confocal imaging (Fig. 2d). To ascertain bona fide morphological phenotypes that stem from the inducible targeted disruption of the $T g \mathrm{Vps} 35$ gene, we complemented this mutant with full-length cMyc-tagged $\mathrm{TgVps} 35$ (Comp-iKoTgVps35), which was introduced in the uracil phosphoribosyl transferase locus, as this gene is known to be non-essential for parasite survival ${ }^{23}$. The iKoTgVps35 mutants were severely impaired in their ability to invade host cells (Fig. 3a) and did not form plaques after multiple rounds of host cell invasion and lysis (Fig. 3b). Complementation of the iKoTgVps35 mutant that allows obtaining Comp$\mathrm{iKoTgVps} 35$ parasite lines restored the ability of these complemented mutants to efficiently reinvade host cells (Fig. 3a), yielding normal plaque sizes in the presence of ATc similar to those of parental RH TaTi parasites (Fig. 3b). These later observations demonstrate that the lack of host cell invasion and the subsequent inability of the iKO mutants to establish several rounds of cell lysis and reinvasion are directly linked to the depletion of $\mathrm{TgVps} 35$ and the absence of functional retromer complex in these mutants, thus excluding pleotropic and non-specific phenotypes.

To examine the role of $T g \mathrm{Vps} 35$ in Toxoplasma infection in vivo, mice were infected with lethal doses of $\mathrm{iKoTgVps} 35$, Comp-iKoTgVps35 or parental parasites followed by $\mathrm{TgVps} 35$ suppression in vivo by providing $\mathrm{ATc}$ in the drinking water. Strikingly, the ATc-treated mice inoculated with iKoTgVps35 survived, whereas animals inoculated with iKoTgVps35 but not treated with ATc succumbed to the infection by day 9 (Fig. 3c). Mice infected with Comp-iKoTgVps35 mutants and the parental strains succumbed to the infection regardless of the initiation of ATc treatment (Fig. 3c). It should be mentioned that $\mathrm{RH}$ TaTi background was genetically attenuated in virulence compared with the parental and wild-type $\mathrm{RH}$ strain, thus allowing challenging mice with sub-lethal parasite doses. When mice were inoculated with sub-lethal doses of these mutants or parental parasites and re-challenged with lethal doses of the wild-type parental RH strain, all iKoTgVps35-infected mice succumbed in a manner similar to the naïve primo-infected animals, whereas those infected with Comp-iKoTgVps35 and the parental strains survived (Fig. 3d). Thus, the conditional ablation of $\mathrm{TgVps} 35$ transformed a $T$. gondii into a complete non-lethal strain of parasites, and furthermore, infection with iKo $\mathrm{TgVps} 35$ parasites does not confer sterile immunity to reinfection, which is also consistent with phenotypic traits previously described for iKoTgSORTLR mutants ${ }^{17}$.

Retromer is essential for parasite integrity. We observed that the disappearance of rhoptries peaks at $24 \mathrm{~h}$ of ATc treatment while micronemes were mostly affected $48 \mathrm{~h}$ after ATc pressure, which also corresponds to the time necessary for the complete depletion of $T g \mathrm{Vps} 35$, as shown by western blots and confocal microscopy in Fig. 2. Following $48 \mathrm{~h}$ of ATc treatment, we also found a complete disorganized morphology with the marked absence of the typical banana-shaped bodies in $\mathrm{TgVps} 35$-depleted mutants using electron microscopy (Fig. 4b), whereas untreated 
a

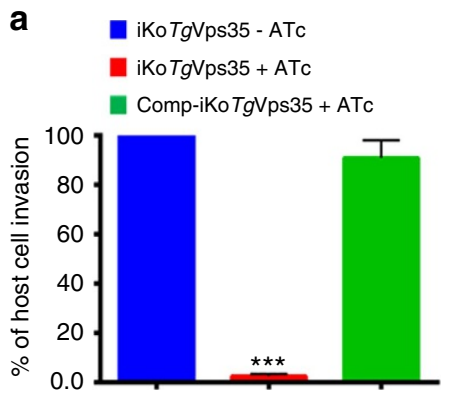

b

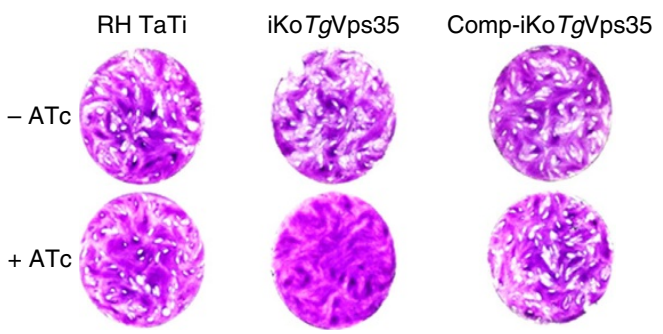

C

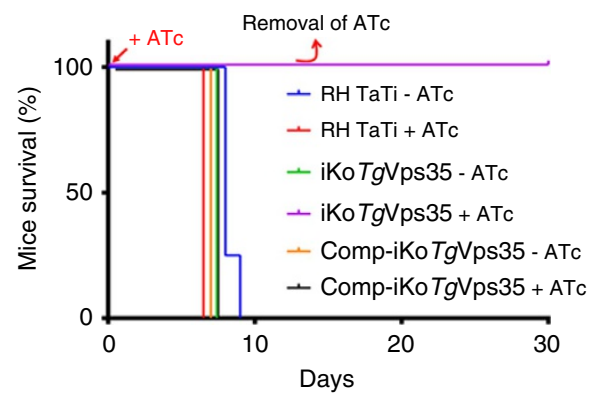

d

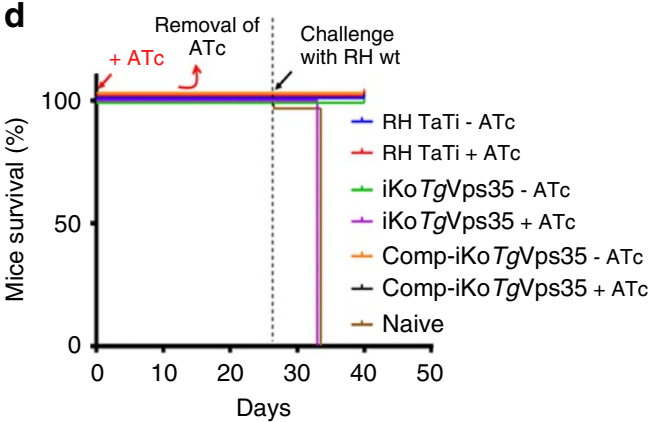

Figure 3 | Resultant phenotypic traits of conditional disruption of $\mathbf{T g V p s 3 5}$ gene. (a) Host cell invasion was assayed in iKoTgVps35 and complemented mutants (Comp-iKoTgVsp35) in the presence and absence of ATc. Bars indicate mean \pm s.d. $(n=3, P<0.001$ by Student's test). (b) Host cell lytic plaques were examined in TgVsp35-deficient mutants, Comp-iKoTgVsp35 and parental RH TaTi parasites in the presence or absence of ATc. (c) Survival of mice infected with lethal doses of TgVsp35-deficient mutants, Comp-iKoTgVsp35 and parental RH TaTi parasites in the presence and absence of ATc in the drinking water. The ATc was removed after 12 days and mice survival was monitored for 30 days post-infection. (d) Avirulent $T g \vee p s 35-d e p l e t e d$ parasites do not confer protective immunity to reinfection with lethal doses of RH wild-type parasites. ATc treatment and mice survival were monitored as above, except that sub-lethal doses of parasites were used during the primo-infection.

a

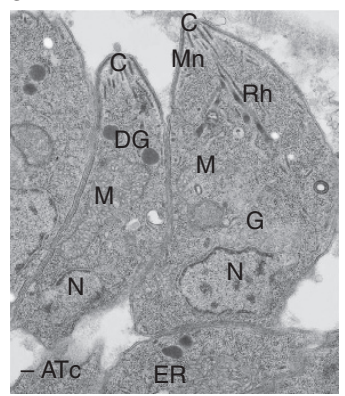

b

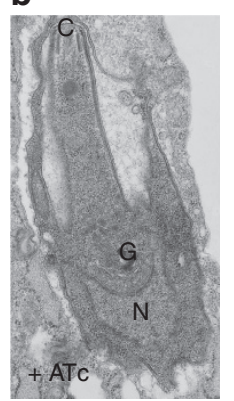

C

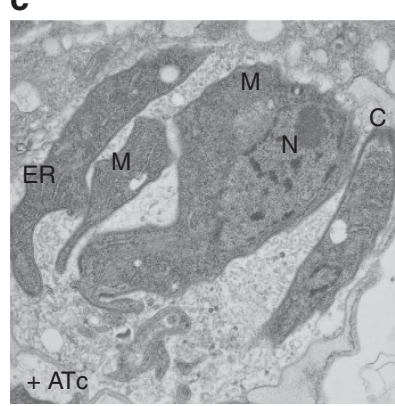

d

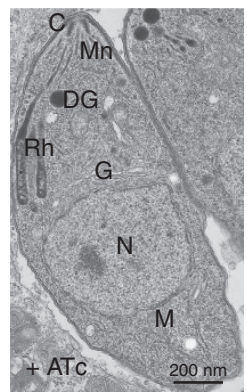

Figure 4 | The $\mathbf{T g V s p 3 5}$ gene is essential for secretory organelle biogenesis and parasite morphology. (a) Transmission electron microscopy showing normal banana-shape morphology of iKoTgVps35 parasites in the absence of ATc. (b) An iKoTgVps35 mutant showing an aberrant morphology. (c) Four replicated daughter iKoTgVsp35 mutants with disorganized body shapes without rhoptries, micronemes and dense granules. (d) Complementation of iKoTgVps35 mutants restored normal parasite morphology with the presence of secretory organelles. C, conoid; DG, dense granules; G, Golgi; $\mathrm{M}$, mitochondria; Mn, micronemes; N, nucleus; Rh, rhoptries. Bar, $200 \mathrm{~nm}$.

iKoTgVps35 parasites appeared structurally normal with all secretory organelles (Fig. 4a). It should be mentioned that the membrane localization of the major glycosyl-phosphatidyl inositol-anchored surface antigens ${ }^{24}$, SAG1 and SAG3 of T. gondii was not impaired by the suppression of $\mathrm{TgVps} 35$ (Fig. 9f), indicating that the traffic to and the integrity of the parasite pellicle were not affected. This aberrant parasite morphology was confirmed in intravacuolar dividing mutants that were also devoid of rhoptries, micronemes and dense granules (Fig. 4c). Complementation of iKoTgVps35 mutants restored the ability of the parasites to form the secretory organelles de novo even in the ATc pressure (Fig. 4d). Using confocal microscopy, we showed that in the absence of rhoptry and microneme organelles, the ROP and MIC proteins were all mis-localized in the cytoplasm as well as in the parasitophorous vacuole of $T g \mathrm{Vps} 35$-depleted mutants, leading to the loss of the typical apical end staining of these proteins (Fig. 5a, lower, left and middle panels, respectively). As expected, the parental parasites normally contained rhoptries and micronemes (Fig. 5a, upper, left and middle panels). In addition, the dense granule GRA1 protein staining that typically surrounds the parasitophorous vacuole (Fig. 5a, upper and right panel) was also altered in $\mathrm{TgVps} 35$-depleted mutants (Fig. 5a, lower and right panel). This later observation is in sharp contrast to the phenotypic traits of iKoTgSORTLR mutants in which dense granule biogenesis and secretion were not affected ${ }^{17}$. We also confirmed that the secretory organelles were correctly localized in complemented $\mathrm{iKoTgVps} 35$ parasites and that the mis-sorting of 
a
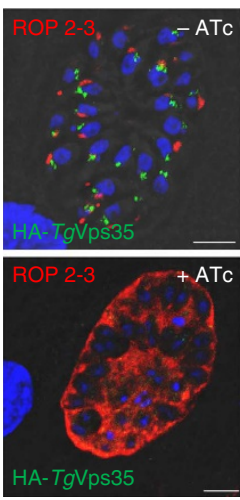
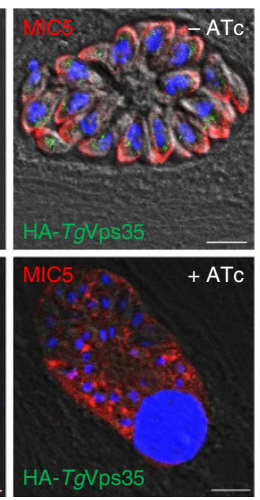
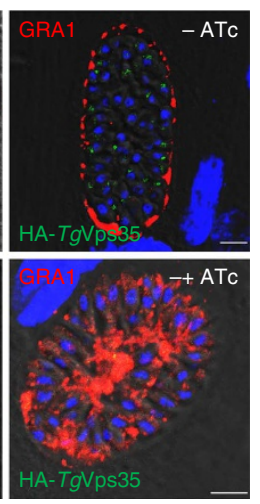

b

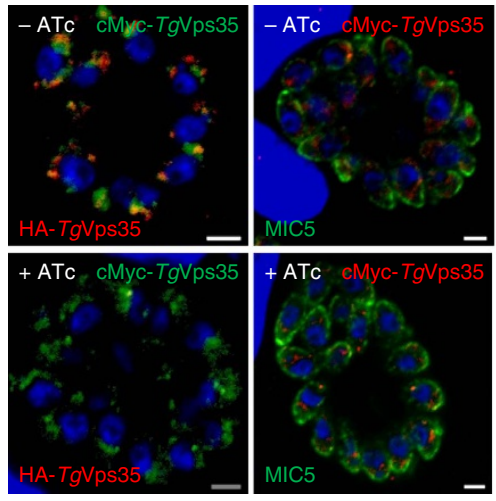

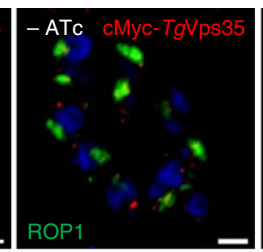
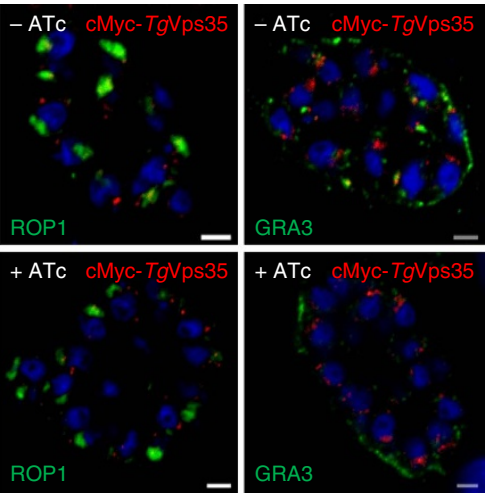

Figure 5 | Conditional ablation of TgVps35 results in mis-sorting of ROP, MIC and GRA proteins. (a) Confocal immunofluorescence microscopy of ROP 2-3, MIC5 and GRA1 proteins in iKoTgVps35 mutants in the presence (lower panels) or absence of ATc (upper panels) for $48 \mathrm{~h}$ using specific antibodies to ROP2-3, MIC5 and GRA1 proteins (see the complete list of antibodies in Supplementary Table 5). Bar, $2 \mu \mathrm{m}$. (b) Confocal immunofluorescence microscopy of MIC5, ROP1 and GRA3 proteins in complemented iKoTgVps35 mutants in the presence (lower panels) or absence of ATc (upper panels) as above. Bar, $2 \mu \mathrm{m}$.
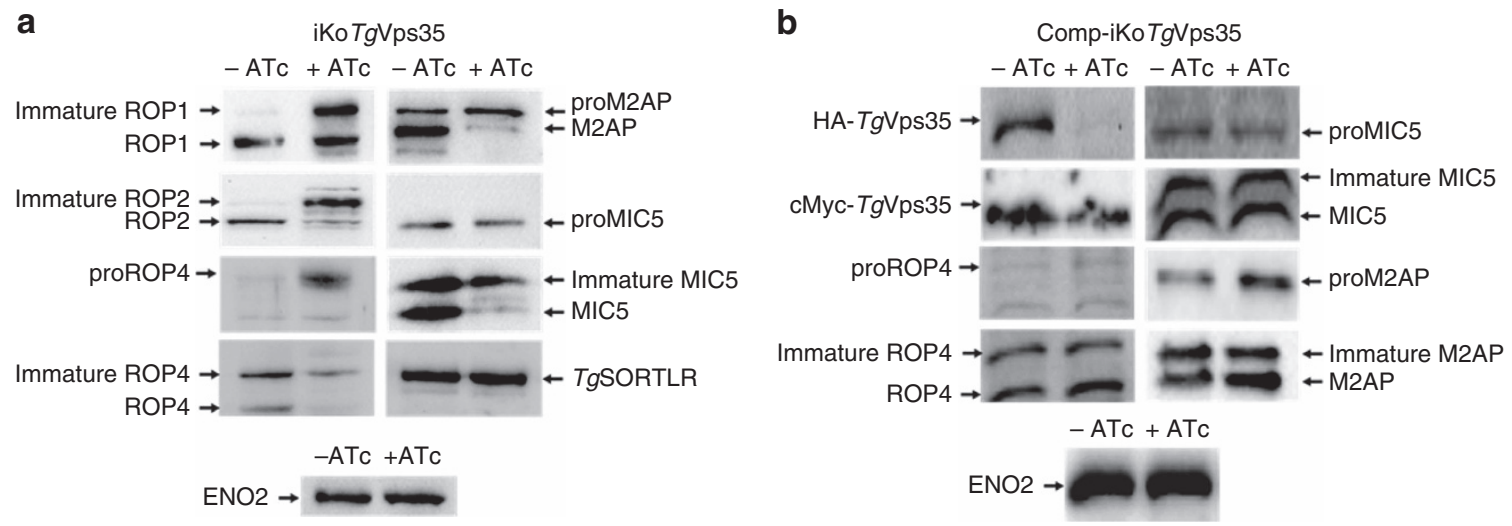

Figure 6 | Disruption of TgVsp35 accumulates unprocessed and immature ROP and MIC proteins. (a) Immunoblots of iKoTg Vps35 mutants probed with specific anti-ROP1, ROP2, proROP4, ROP4, M2AP, proMIC5 and MIC5 antibodies after $48 \mathrm{~h}$ post-infection in the presence or absence of ATc. TgSORTLR and ENO2 protein levels were identical in both ATc-treated and untreated mutants. (b) Immunoblots of complemented iKoTgVps35 mutants probed with specific anti-ROP1, ROP2, proROP4, ROP4, M2AP, proMIC5 and MIC5 antibodies after $48 \mathrm{~h}$ post-infection in the presence or absence of ATc. ENO2 protein levels were identical in both ATc-treated and untreated mutants.

ROP, MIC and GRA proteins was rescued in the presence of ATc using confocal microscopy (Fig. 5b).

In wild-type parasites, formation of rhoptries and micronemes is correlated with proteolytic maturation of ROP and MIC proteins $^{3,25}$. Likewise, this proteolytic maturation was defective in the $T g V p s 35-d e p l e t e d$ mutants compared with the parental strain, leading to the accumulation of unprocessed ROP1, ROP2, ROP4, M2AP and MIC5 (Fig. 6a and Supplementary Fig. 2). Notably, iKoTgVps35 parasites that were not treated with ATc displayed typical proteolytic maturation of the aforementioned ROP and MIC proteins (Fig. 6a and Supplementary Fig. 2). Next, we probed these blots with specific antibodies that recognized the $\mathrm{N}$-terminal pro-peptides of ROP4 and MIC5 and found a significant accumulation of both pro-protein and immature forms in the $T g V p s 35$-deficient mutants (Fig. 6a and Supplementary Fig. 2). In contrast, processing of the receptor $T g$ SORTLR was unchanged in these mutant parasites, suggesting that neither the processing of pre-protein in the endoplasmic reticulum is impaired nor this receptor is subjected to the typical lysosomal-like degradation in the $T g V p s 35$-deficient parasites (Fig. 6a). This later behaviour of $T$. gondii lacking $T g V s p 35$ and retromer functions differs greatly from what is normally observed in the absence of functional retromer complex in other eukaryotes in which the cargo sorting receptors such as sortilin and mannose-6-phosphate receptor are targeted to lysosomes for degradation ${ }^{26-29}$. Since the levels of the control protein, the glycolytic enzyme enolase $\mathrm{ENO} 2$, were similar between mutant and parental parasites, we speculated that both pro-ROP and pro-MIC specifically accumulated in $\mathrm{iKoTgVps} 35$ mutants as a consequence of conditional disruption of $\mathrm{TgVps} 35$ functions, which are clearly recovered in the presence of $i K o T g V p s 35$ complementation as cMyc- $\mathrm{TgVps} 35$ is able to fully restore proteolytic processing and maturation of ROP and MIC proteins under ATc pressure (Fig. 6b). Taken together, these results suggest that retromer-mediated recycling is likely required to deliver and maintain one or more proteases that process pro-ROP and pro-MIC proteins, a proteolytic processing that is a key parameter for secretory organelle formation and host infectivity by $T$. gondii.

Secretory organelle biogenesis depends on retromer. The cellular location of $T g \mathrm{Vps} 35, \mathrm{TgVps} 26$ and $\mathrm{TgVsp} 29$ of the retromer complex with the ELC markers proM2AP and vacuolar protein 1 (Fig. 1d), as for Rab5 or Rab7, prompted us to investigate the outcome of $\mathrm{TgVps} 35$ depletion on TgSORTLR, which also co-distributes in the parasite with these two 

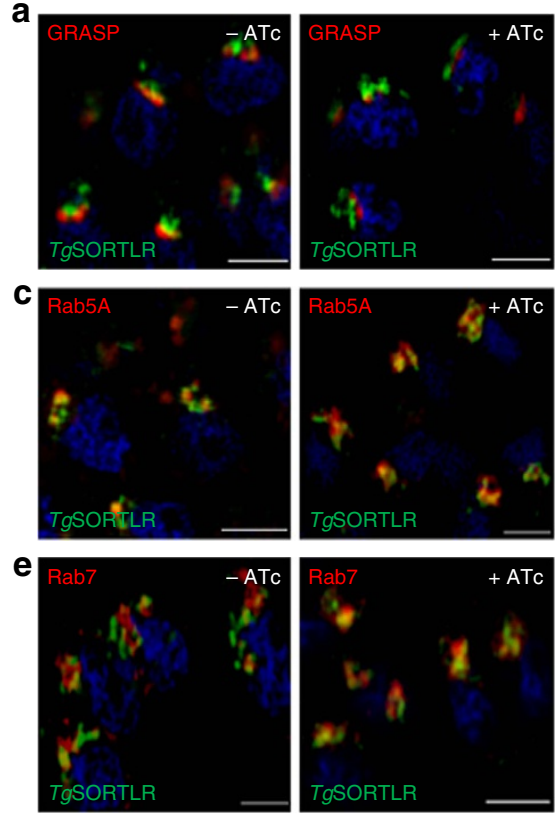
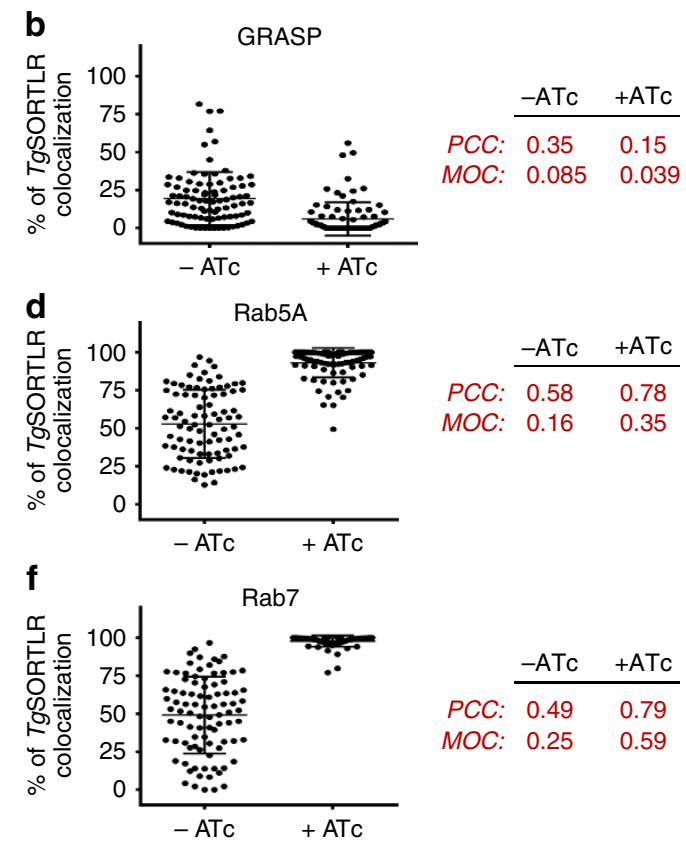
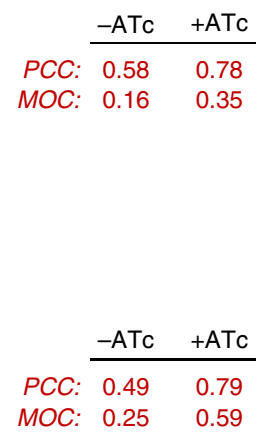

Figure 7 | Recycling of TgSORTLR is blocked in Rab5A- and Rab7-decorated ELCs. (a) SIM imaging of the co-distribution of TgSORTLR with Golgi marker GRASP in iKoTgVps35 mutants in the absence or presence of ATc. Bar, $2 \mu \mathrm{m}$. (b) Quantification of the co-distribution of TgSORTLR with Golgi marker GRASP in iKoTgVps35 mutants by SIM in the absence or presence of ATc. Bars indicated the mean $n=90$ parasites from 7 to 8 vacuoles \pm s.d., $P<0.0001$. (c) Co-distribution of TgSORTLR with the marker of early endosome Rab5A in iKoTgVps35 mutants grown in the presence or absence of ATc and treated for SIM imaging as above. Bar, $2 \mu \mathrm{m}$. (d) Quantification of TgSORTLR co-distributing with the marker of early endosome Rab5A in iKoTgVps35 mutants by SIM in the absence or presence of ATc. Bars indicated the mean $n=90$ parasites from 7 to 8 vacuoles \pm s.d., $P<0.0001$. (e) Co-distribution of TgSORTLR with the marker of late endosome marker Rab7 in iKoTgVps35 mutants by SIM imaging as above. Bar, $2 \mu \mathrm{m}$. (f) Quantification of TgSORTLR co-distributing with the marker of late endosome marker Rab7 in iKoTgVps35 mutants by SIM in the absence or presence of ATc. Bars indicated the mean $n=90$ parasites \pm s.d., $P<0.0001$. The Pearson correlation coefficient (PCC) and the Mander's overlap coefficient (MOC) used to quantify the degree of colocalization between the red and green fluorophores were shown on the right.

small GTPases ${ }^{17}$. Using high-resolution structured illumination microscopy (SIM), we monitored the discrete compartments that contained TgSORTLR (Fig. 7) by co-labelling Golgi apparatus marker with the Golgi reassembly stacking protein (GRASP)RFP, the early endosome with HA-Rab5A, and the late endosome with HA-Rab7. While endogenous TgSORTLR (up to 20\%) colocalized with GRASP-RFP in the Golgi compartment (Fig. 7a, left panel) in the parental strain, the co-distribution drastically decreased to a marginal level in $T g V p s 35$-deficient mutants (Fig. 7a, right panel and Fig. 7b). Consequently, we observed significantly increased colocalization of endogenous TgSORTLR with Rab5A and Rab7-positive ELC in TgVps35-depleted mutants (Fig. 7c,e; right panels). Quantitation of this co-distribution in $T g \mathrm{Vps} 35$-deficient mutants indicated that $90 \%$ of TgSORTLR localized with Rab5A (Fig. 7d) and that up to $99 \%$ of endogenous TgSORTLR was colocalized with Rab7-positive ELC (Fig. 7f). In comparison, only $60 \%$ (Rab5A) and 50\% (Rab7) of TgSORTLR was colocalized with the Rab GTPase-labelled ELC in parasites that were not treated with ATc (Fig. 7d,f). Biochemical data also supported the notion that only TgVps26 interacts with Rab7 in a GTP-dependent manner, while none of these three subunits of the T. gondii core retromer partnered with Rab5B or Rab11B in the presence of GTP (Supplementary Fig. 3). Clearly, these data demonstrate that the retromer complex drives TgSORTLR recycling from Rab7-positive ELC to the TGN, thus sustaining another round of protein transport for proper secretory organelle biogenesis.

$T g \mathrm{HP} 12$ is a parasite-specific retromer-associated partner. The conditional disruption of the $T g V p s 35$ gene strongly suggests that the retromer complex is likely involved in other functions, such as controlling parasite shape in addition to secretory organelle biogenesis. In our quest to discover other roles for the retromer complex in T. gondii, we sought to decipher the functions of some parasite-specific proteins also named HP that were identified in the interactome (Fig. 1c and Supplementary Data 1). Towards this goal, we searched by bioinformatics for striking sequence features that could potentially define retromer-associating proteins among these parasite-specific HP proteins. We identified a typical type I transmembrane $\mathrm{TgHP} 12$ protein that harbours a putative coiled-coil region downstream of the transmembrane segment (Supplementary Fig. 4a). These structural features are conserved in $\mathrm{TgHP} 12$ homologues in all tested parasites across the Apicomplexa phylum (Fig. 8a). Potential relationships were identified between the TgHP12 helical region and two helical structures present in rabenosyn-5 and FIP2 (Supplementary Fig. $4 \mathrm{~b}$ ), which are known to be involved in the interaction with the Rab GTPases ${ }^{30,31}$, and also with the coiled-coil region of syntaxin, which shares typical heptad repeats with TgHP12 (Supplementary Fig. 4b).

Because these similarities represent only short segments, statistical analysis was unable to establish a significant relationship with any of the proteins. As a result, we have not further investigated the significance of these possible structural features, but instead, we determined the molecular relationships between $\mathrm{TgHP} 12$ and the retromer complex by knock-in $\mathrm{TgHP} 12-\mathrm{cMyc}$ into iKoTgVsp35 mutants. These experiments revealed that TgHP12 co-localizes with TgVps35-HA and TgSORTLR (Fig. 8b, upper panels) but not with cathepsin L or M2AP, two markers of the ELC (Fig. 8b, lower panels), suggesting that TgHP12 is a resident protein of both the Golgi and TGN compartments. Likewise, mass spectrometry was used to 
a

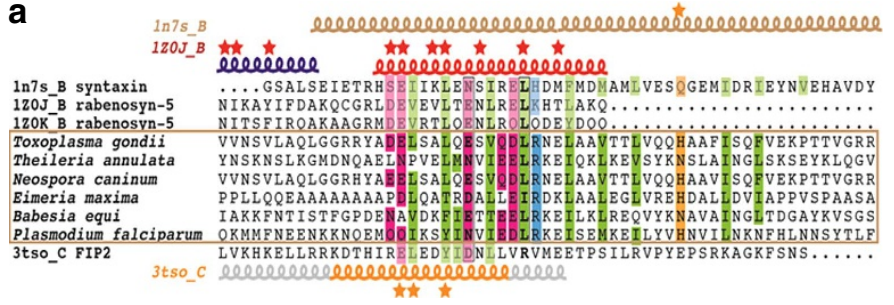

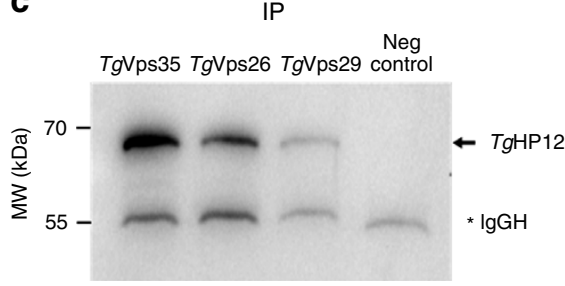

b

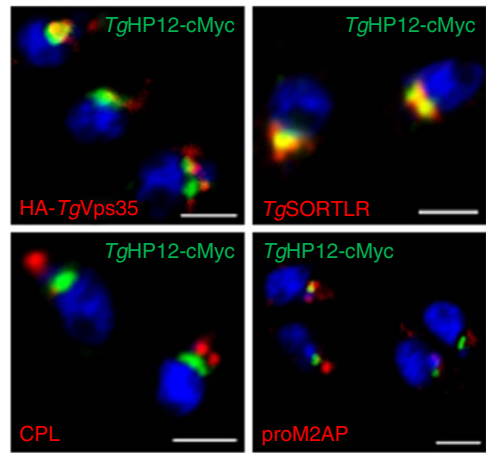

d

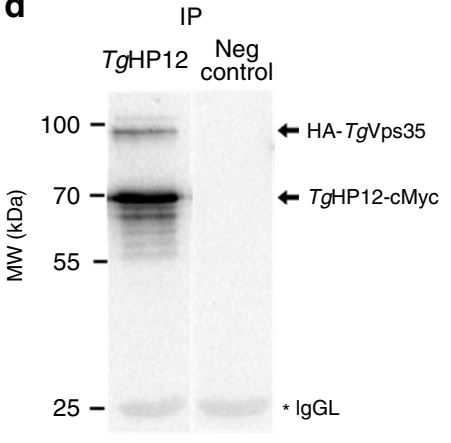

e

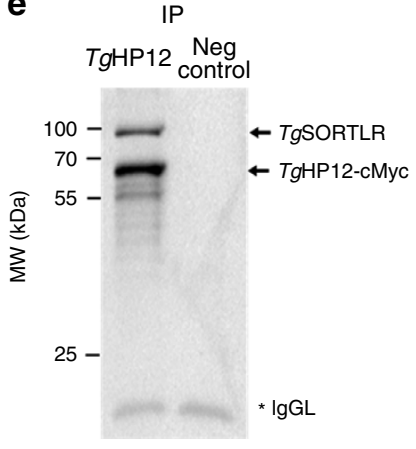

Figure 8 | TgHP12 is a novel parasite-specific retromer-associating partner. (a) Alignment of the helical region in TgHP12 protein and in orthologues from apicomplexan parasites with human syntaxin (pdb 1n7s_B, syntaxin), rabenosyn-5 (1z0j_B and 1zOk_B), and FIP2 (pdb 3tso_C). Secondary structures are reported above and below the alignment. Stars indicate amino acids involved in Rab binding (rabenosyn-5 and FIP2) and the conserved glutamine of syntaxin that participates in the ionic central layer of SNARE complexes. Genbank identifier (gi) and N- and C-terminal limits are as follows: Theileria annulata (85000999, aa 239-297), Neospora caninum (401395596, aa 42-100), Eimeria maxima (557188226, aa 283-342), Babesia equi (510902511, aa 253-311) and Plasmodium falciparum (583212139, aa 375-433). Also see Supplementary Fig. 4. (b) Confocal immunofluorescences showing co-distribution of $\mathrm{TgHP} 12$ protein with $\mathrm{TgVps} 35$ protein, and TgSORTLR protein (upper panel) while no colocalization was detected with cathepsin L (CPL) or proM2AP protein. Mouse or rabbit polyclonal anti-cMyc antibodies were used in addition to rat anti-TgSORTLR, rabbit anti-HA, anti-CPL and proM2AP antibodies. Bar, $2 \mu \mathrm{m}$. (c) Reverse immunoprecipitation using total detergent extract proteins from the knock-in TgVps35-HA, TgVps29-cMyc or TgVps26-HA parasites demonstrated that all three components of the core retromer complex can be pulled down by TgHP12. IgG $\mathrm{h}_{\mathrm{h}}$ IgG heavy chain; IP, immunoprecipitations; Neg control, negative control using naïve sera; molecular weights $(\mathrm{kDa})$ were shown on left. (d) TgHP12-cMyc and TgVps35-HA proteins were concomitantly co-immunoprecipitated from total detergent protein extracts from iKoTgVps35 parasites in which TgHP12-cMyc protein was expressed by knock-in strategy. The blots were probed with rabbit anti-cMyc and anti-HA. IgG, IgG light chain; IP, immunoprecipitations; Neg control, negative control using naïve sera; molecular weights $(\mathrm{kDa})$ were shown on left. (e) TgHP12-cMyc and TgSORTLR proteins were concomitantly co-immunoprecipitated from parasites total detergent protein extracts from iKoTgVps35 parasites in which TgHP12-cMyc protein was expressed by knock-in strategy. The blots were stained with rabbit anti-cMyc and rat anti-TgSORTLR. IgG $G_{L}$ IgG light chain; IP, immunoprecipitations; Neg control, negative control using naïve sera; molecular weights $(\mathrm{kDa})$ were shown on left.

demonstrate that $T g \mathrm{HP} 12$ specifically pulled down $T g \mathrm{Vps} 35$, $T g V p s 29$ and $T g V p s 26$ in addition to TgSORTLR (Supplementary Table 2). These interactions were confirmed by reverse immunoprecipitations in which $T g V s p 35, T g V s p 29$ and $T g V p s 26$ also specifically pulled down TgHP12 protein (Fig. 8c). Furthermore, we confirmed that the eluates of $T g \mathrm{HP} 12$ also contained HA-TgVps35 (Fig. 8d) and TgSORTLR (Fig. 8e) by western blots. To obtain additional insight into the functions of $T g H P 12$, we disrupted this gene using the CRISPR-Cas9 strategy (Supplementary Fig. 4c). We confirmed the efficient disruption of $\mathrm{TgHP} 12$ gene, as no $\mathrm{TgHP} 12$ protein was detected in these mutants using rat polyclonal antibodies that we specifically raised and purified against the recombinant $T g \mathrm{HP} 12$ protein (Supplementary Fig. 4d, first and left of upper panels). As expected, this protein was normally expressed in wild-type parasites (Supplementary Fig. 4d, red, first and left of lower panels). Since no deleterious effects in rhoptries, micronemes or dense granules occurred following CRISPR-Cas9 disruption of TgHP12, we conclude that this type I transmembrane protein is likely involved in functions distinct from those described for TgSORTLR. In line with this hypothesis, proteomic analysis indicated that TgHP12 binds to other partners (Supplementary Data 3), suggesting a possible role in alternative trafficking pathways or in the regulation of other functions as a retromer-associated partner.
Retromer maintains a parasite transporter at the membrane. The generation of $\mathrm{iKoTgVps} 35$ mutants that are deficient in retromer-mediated transport allows us to investigate the recycling mechanisms that deliver and maintain transmembrane proteins at the parasite membrane. We also used bioinformatics to search for candidate multi-spanning transmembrane proteins among the HP identified in the retromer interactome. We discovered that $\mathrm{TgHP} 03$ exhibits the topology and positions of 12 transmembrane helices that were predicted by Phyre2 alignments ${ }^{32}$ to align with known three-dimensional structures of several members of the major facilitator superfamily (MFS), suggesting that $\mathrm{TgHP} 03$ may belong to this superfamily (Fig. 9a). These membrane transporters facilitate movement of a wide range of small substrates such as metabolites, oligosaccharides, amino acids, oxyanions and drugs that were all transported by MFS across the cell membranes ${ }^{33}$. Even though a significant relationship with the MFS can be established, no direct link with one specific MSF member was determined by bioinformatics. To assess the functional links between $\mathrm{TgHP} 03$ and the retromer complex, we first examined its sub-cellular localization by detecting a cMyc epitope-tagged version in the iKoTgVps35 mutants. In the absence of ATc, TgHP03-cMyc displayed homogenous membrane staining on both extracellular (Fig. 9b,c, left upper panel) and intracellular (Fig. 9c, right upper panel) parasites, 
a

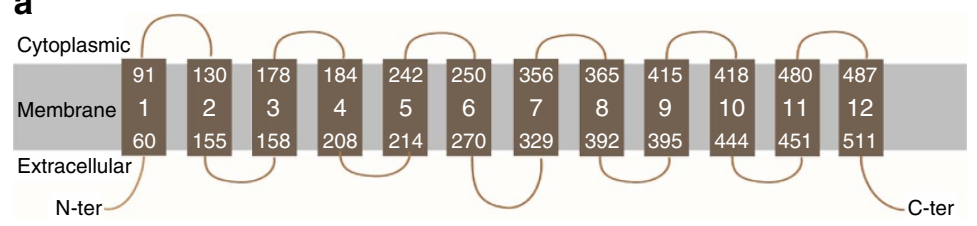

d

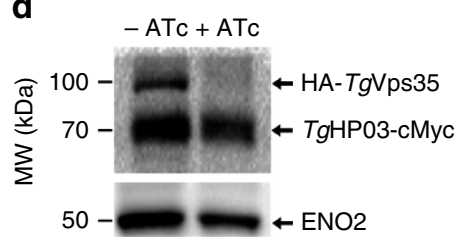

e

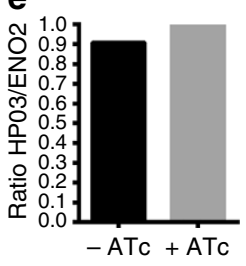

b

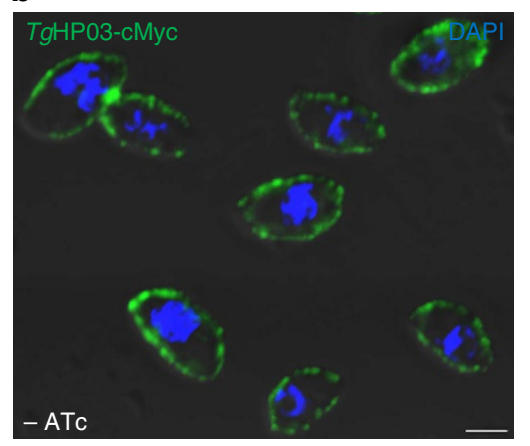

C

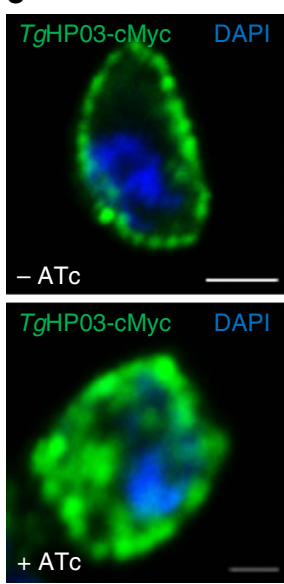

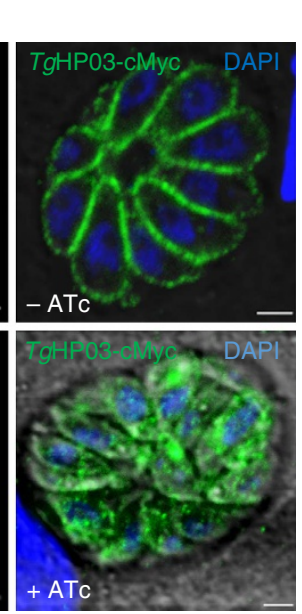

$\mathbf{f}$
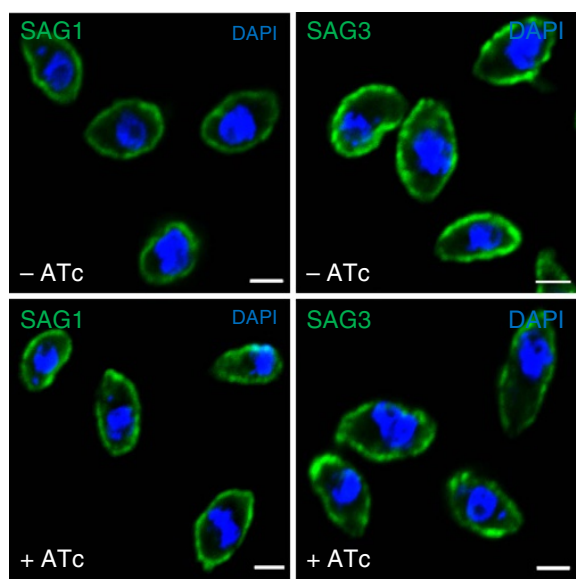

Figure 9 | Retromer is required to maintain TgHP03 in the parasite membrane. (a) Schematic representation of TgHPO3 topology inside the parasite membrane. (b) Confocal imaging of extracellular parasites with knock-in TgHPO3-cMyc in the iKoTgVps35 mutants. Immunofluorescence assay (IFA) was performed in the absence of ATc and detergent permeabilization. Nuclei of the parasites as stained with 4', 6-diamidino-2-phenylindole (DAPI; blue). Bar, $2 \mu \mathrm{m}$. (c) Magnified image of an extracellular parasite expressing TgHPO3-cMyc protein in the iKoTgVps35 mutants grown in the absence of ATc and detergent permeabilization (upper, left panel); intracellular parasites expressing TgHP03-cMyc protein in the iKoTgVps35 genetic background. IFA was performed in the absence of ATc and in the presence detergent permeabilization (upper, right panel); magnified image of an intracellular parasites expressing TgHPO3-cMyc protein in the iKoTgVps35 genetic background in the presence of ATc and detergent permeabilization (lower, left panel) and intracellular parasites expressing TgHPO3-cMyc protein in the iKoTgVps35 genetic background in the presence of ATc and detergent permeabilization (lower, right panel). Bar, $2 \mu \mathrm{m}$. (d) Immunoblots of parasites expressing TgHP03-cMyc protein in the knock-in iKoTgVps35 mutants grown in the presence or absence of ATc. ENO2 was used as a loading control. Molecular weights (kDa) were shown on left. (e) Quantification of TgHPO3 levels in these parasites expressing TgHPO3 protein in the knock-in iKoTgVps35 mutants that were grown in the absence or presence of ATc. (f) The surface localization of glycosylphosphatidyl inositol (GPI)-anchored SAG1 and SAG3 were determined in iKoTgVps35 mutants in the presence or absence of ATc using monoclonal antibodies specific to SAG1 and SAG3. Bar, $2 \mu \mathrm{m}$.

as expected for transmembrane transporters. $\mathrm{TgVps} 35$ suppression by ATc led to a mis-localization and enhanced accumulation of $\mathrm{TgHP} 03$ in intra-cytoplasmic vesicular structures in both extracellular (Fig. 9c, left lower panel) and intracellular mutants (Fig. 9c, right lower panel), thus leading to a decrease of $\mathrm{TgHP} 03$ at the parasite surface. Since ATc treatment did not affect the levels of $T g \mathrm{HP} 03-\mathrm{cMyc}$ protein (Fig. 9d,e), it is likely that no $\mathrm{TgHP} 03$ protein degradation may occur on suppression of $\mathrm{Tg} \mathrm{Vps} 35$-mediated endocytic recycling. This observation is also consistent with the absence of TgSORTLR degradation following suppression of $\mathrm{TgVps} 35$ (Fig. 6a). Again, the accumulation of mis-sorted cargo in intra-cytoplasmic vesicles in T. gondii is also in sharp contrast to transmembrane transporter degradation occurring via lysosomes in mammalian cells ${ }^{34}$. Interestingly, we found that the surface localization of the glycosylphosphatidyl inositol-anchored major surface antigens, SAG1 and SAG3 of T. gondii was not influenced by the suppression of $\mathrm{TgVps} 35$ (Fig. 9f), suggesting that only the sorting of multi-spanning transmembrane proteins may be influenced by retromer-dependent endocytic recycling. Together, our data identify a role for $\mathrm{TgVsp} 35$ in the endosome-to-plasma membrane sorting of multi-spanning transmembrane transporter cargo and provide the first evidence for the mechanistic role of $\mathrm{TgVsp} 35$ in the process linked to retromerdependent endocytic recycling.

\section{Discussion}

Nascent apical organelles require proficient cell trafficking to fulfil their critical role during invasion and intracellular development of $T$. gondii. Here, we show that retromer-mediated recycling is essential for secretory organelle biogenesis, parasite morphology and maintenance of a transmembrane transporter at the parasite membrane. The retromer complex was first identified in yeast and mammals as a heteropentameric complex typified by a cargoselective complex that was built around the Vps35-Vps29-Vps26 trimer and a dimer of different sorting nexins ${ }^{28,35-38}$. Our previous findings that TgSORTLR receptor is involved in the recruitment of Vps 26 and Vps35 homologues prompted us to investigate how the retromer complex regulates retrograde transport as well as other functions in T. gondii. Towards this goal, we characterized the retromer interactome that includes $T g \mathrm{Vps} 35, T g \mathrm{Vps} 29$ and $T g \mathrm{Vps} 26$ proteins. We failed to identify homologues of nexins (SNX) in the immunoprecipitates of T. gondii using all three retromer components, confirming that no SNX proteins can be pulled down in association with the retromer cargo-selective trimer ${ }^{39}$. However, we also noticed the paucity of nexin-like proteins in the genome databases of T. gondii, and this may also explain our inability to pull down recognizable nexin-like proteins in the parasite. Rather, we identified a singular retromer complex typified by the $T g \mathrm{Vps} 35-T g \mathrm{Vps} 29-T g \mathrm{Vps} 29$ trimer. This complex interacts 
with TgSORTLR, $N$-ethylmaleimide-sensitive protein, Rab5B, Rab11B and the TBC1D5A homologue, a member of the Rab7-GTPase-activating proteins known to negatively regulate the retromer complex through Rab7 dissociation from endosomal membranes $^{22}$. However, we cannot rule out the possibility that some parasite-specific proteins may act as SNX and Bin/Amphyphysin/Rvs domain-containing proteins that are required for recruitment of the retromer complex to endosome membranes, membrane curvature and tubulation ${ }^{27,35}$. Furthermore, we demonstrated that TgSORTLR recycling from the ELC to the TGN is severely compromised in $\mathrm{TgVps} 35$ mutants, suggesting that the retromer complex functions in endocytic recycling in $T$. gondii. In contrast to yeast and human cells in which Rab7-Vps35 interaction is necessary for binding to endosome membranes 37,38 , we found an unconventional $T g \mathrm{Rab} 7-T g \mathrm{Vps} 26$ interaction, a singular feature previously described in Entamoeba histolytica ${ }^{40}$.

In addition, we describe additional evidence for the phenotypic links between an absence of proteolytic processing of ROP/MIC proteins, mis-sorting of their unprocessed forms, and loss of rhoptry and microneme organelles. Our hypothesis is that TgSORTLR, in addition to acting as a sorting receptor for $\mathrm{ROP} / \mathrm{MIC}$ proteins, delivers proteases, which are involved in the processing of pro-proteins, to secretory organelles.

The retromer interactome also includes several parasite-specific proteins. We first described $T g \mathrm{HP} 12$ as a novel type I transmembrane protein with helical regions that share potential similarities with Rab-binding or SNARE-like domains. We showed that TgHP12 localizes to the TGN and physically interacts with the core retromer complex $T g \mathrm{Vps} 35-\mathrm{TgVps} 29-$ $T g \mathrm{Vps} 26$ as well as with $\mathrm{TgSORTLR}$. Genetic ablation of TgHP12 using CRISPR-Cas9 indicates that its function is neither essential nor related to secretory organelle biogenesis. Instead, proteomic analyses revealed other unrelated secretory organelle proteins whose functions remain to be determined. Our current study also describes for the first time in apicomplexan parasites, the endocytic recycling of the multi-pass transmembrane transporter $\mathrm{TgHP} 03$, which belongs to the MFS, a class of membrane transport proteins that facilitate movement of small solutes across cell membranes such as drugs, metabolites, oligosaccharides and amino acids in response to chemiosmotic gradients ${ }^{41}$. $\mathrm{TgHP03}$ accumulates in endocytic vesicles that are detected in the cytoplasm of $\mathrm{TgVps} 35$-deficient mutants, indicating that maintenance of $\mathrm{TgHPO} 3$ at the surface of $T$. gondii requires endocytic recycling from endosomes to the plasma membrane. Clearly, our study underscores the wide range of possible cargo molecules that are recycled by the retromer complex in light of the numerous identified transmembrane proteins that require future examination. A global analysis of retromer-mediated cargo per se will aid the delineation of the diverse metabolite and metal ion transporters required to maintain parasite nutrient homeostasis and intracellular replication.

It is also worth noting that the absence of $T g \mathrm{Vps} 35$ has negatively impacted the biogenesis of dense granules and body morphology in addition to that of rhoptries and micronemes, whereas TgSORTLR functions are restricted to rhoptry and microneme formation ${ }^{17}$. This disorganization of parasite body shape in the absence of $\mathrm{TgVsp} 35$ suggests a possible additional role of the retromer in regulating cytoskeleton and endosome functions as previously described in mammalian cells ${ }^{42,43}$. However, the sorting nexins that are involved in this process in mammalian cells are not presently identified in $T$. gondii. Alternatively, we cannot rule out the possibility that the parasite-specific HP or other retromer-associated proteins could play this novel function that controls body morphology in T. gondii.

In conclusion, we demonstrated that the retromer complex of T. gondii is a major endosomal recycling hub required for sorting different cargo proteins that regulate diverse functions vital for parasite survival, as indicated in the our model presented in Fig. 10. This model also supports the notion that the retromer complex drives TgSORTLR recycling from Rab7-positive ELC to the TGN, thus sustaining another round of protein transport for proper secretory organelle biogenesis (Fig. 10a). In contrast to higher eukaryotes, in which an increase of lysosome degradation of the glucose transporter and other cargo sorting proteins,
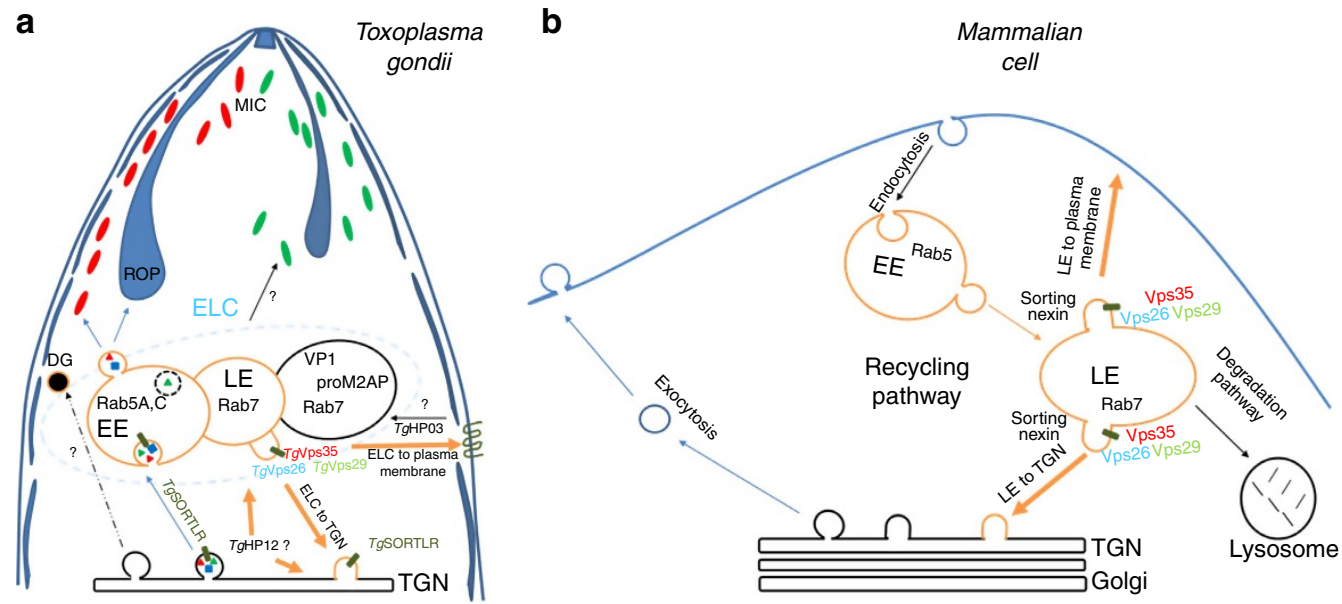

Figure 10 | Model of retromer-mediated recycling of diverse sorting cargoes in $\mathbf{T}$. gondii versus mammalian cells. No recognizable sorting nexins, Bin/Amphyphysin/Rvs (BAR)-containing domain proteins or lysosomal-like protein degradation have been identified in T. gondii (a), in contrast to the situation in mammalian cells (b). Instead, the retromer-dependent recycling is essential for secretory organelle formation and parasite shape. We propose a model suggesting that Rab7 is the key small GTPase, which is involved in the endocytic recycling of TgSORTLR from endosomes to TGN for another round of ROP and MIC transport and secretory organelle biogenesis. We further raised the possibility that in contrast to mammalian cells, $T$. gondii lysosomal-like organelles only promote proteolytic maturation of proteins destined to secretion and that the endosomal system is adapted for organellar discharge of virulence-like factors required for the intracellular lifestyle of the parasite. Moreover, we provide the first evidence that a multiple ligand transmembrane transporter $\mathrm{TgHPO} 3$ is maintained at the surface of $T$. gondii through endocytic recycling from endosomes to the plasma membrane. BAR, Bin/Amphyphysin/Rvs; MIC, microneme; ROP, rhoptry; TgHPO3, T. gondii hypothetical protein 03. 
sortilin and mannose-6-phosphate receptor occurred in the absence of $\mathrm{Vps} 35$ (refs 28,34), the disruption of $\mathrm{TgVsp} 35$ did not affect TgSORTLR and TgHP03 degradation. This later observation also supports our model that in contrast to mammalian cells (Fig. 10b), T. gondii lysosomal-like organelles $^{11,44,45}$, only promote proteolytic maturation of proteins destined to secretion and that the endosomal system is adapted for organelle biogenesis and discharge of factors required for the intracellular lifestyle of the parasite (Fig. 10a). Our findings are expected to advance our understanding of endocytic recycling by $T$. gondii, highlighting the identity of unconventional endosomal factors, and this new knowledge may ultimately reveal new targets for managing toxoplasmosis.

\section{Methods}

Growth of host cells and parasite strains. T. gondii $\mathrm{RH}$ wild-type strain and its derivative RH $\Delta$ ku80 (ref. 46) or RH TaTi (ref. 47) strains were grown in human foreskin fibroblasts (HFF; from ATCC, USA) as described ${ }^{17}$. Plaque size assays were performed using HFF cells infected with 250 parasites in the presence or absence of $1.5 \mathrm{\mu g} \mathrm{ml}^{-1}$ of ATc for 6 days followed by crystal violet staining. All stained coverslips were imaged with an Axioplan microscope (Zeiss).

\section{Generation of knockout and knock-in T. gondii strains. All primers used for PCR} are listed in Supplementary Table 3. The conditional iKoTgVps35 mutants were generated in the RH TaTi strain using the pG13-D-T7S4 plasmid, which contains $2 \mathrm{~kb}$ of $5^{\prime}$ and $3^{\prime}$ genomic DNA and the dihydrofolate reductase (DHFR) gene for pyrimethamine selection. After transfection of $5 \times 10^{6}$ parasites with $50 \mu \mathrm{g}$ of linear plasmid, a stable line was cloned by limiting dilution. For complementation, iKoTgVps35 mutants were transfected with $50 \mu \mathrm{g}$ of plasmid containing the full-length cMyc-tagged $\mathrm{TgVps} 35$ gene inserted into the uracil phosphoribosyl transferase locus. Stable transgenic and cloned parasites were selected with $5 \mu \mathrm{M}$ 5-fluoro-2'-deoxyuridine. Transgenic $T g \mathrm{Vps} 35-\mathrm{HA}, T g \mathrm{Vps} 26-\mathrm{HA}$ and $T g \mathrm{Vps} 29$ cMyc parasites were generated with a knock-in strategy with DNA fragments of 2.1, 2.1 and $1.8 \mathrm{~kb}$, respectively, cloned upstream of the stop codon from TGGT1_242660, TGGT1_263500 and TGGT1_252490 genes. DNA sequences were cloned into the pLIC-HA-DHFR and pLIC-cMyc-DHFR plasmids ${ }^{46}$.

Tachyzoites $\left(5 \times 10^{6}\right.$ parasites $)$ of the RH $\Delta \mathrm{Ku} 80$ strain were transfected with $25 \mu \mathrm{g}$ of linearized plasmids. Transgenic parasites expressing cMyc-tagged TgHP03 (TGGT1_240810) and TgHP12 (TGGT1_294220) were also generated as described above. All plasmids used in this study are listed in Supplementary Table 4.

Transient transformation and Cas9-mediated gene disruption. The plasmid pTOXO_Cas9-CRISPR (see map in Supplementary Fig. 4, panel c) corresponds to pUC57 carrying the C-terminally HA/GFP tagged S. pyogenes Cas 9 gene $\mathrm{e}^{48}$ fused to 2 nuclear localization sequences expressed under the control of the TUB8 promoter as well as the TgU6 promoter driving the gRNA. Twenty mers-oligonucleotides corresponding to specific gene of interest were cloned using Golden Gate strategy ${ }^{49}$. The $\mathrm{ccdB}$ positive-selection marker acts by killing the background of cells with no cloned DNA. The plasmid was synthesized and fully sequenced by GenScript (Singapour).

\section{Confocal microscopy. Extracellular or intracellular parasites were fixed with} $4 \%$ paraformaldehyde in phosphate-buffered saline for $20 \mathrm{~min}$ and processed as described ${ }^{17,50}$ using the indicated antibodies. The sources, origins and dilutions of all antibodies used for immunofluorescence assays were listed in Supplementary Table 5. Samples were observed with a Zeiss Confocal or Apotome microscope, and images were processed using ZEN software (Zeiss).

Structured illumination microscopy. SIM was used to obtain high-resolution images using an ElyraPS1 microscope system (Zeiss) with a $\times 100$ oil immersion lens (alpha Plan Apochromat $\times 100$, NA 1.46, oil immersion). The resolution was measured using beads with a diameter of $100 \mathrm{~nm}$ (Tetraspeck multicolor). The point spread function was calculated using the metroloJ plugin (ImageJ, NIH), which gave an $x-y$ resolution of $125 \mathrm{~nm}$ and a $z$ resolution of $500 \mathrm{~nm}$. A voxel size of $0.050 \times 0.050 \times 0.150 \mu \mathrm{m}^{3}$ was used for the measurement. Three lasers $(405,488$ and $561 \mathrm{~nm}$ ) were used for excitation. SIM images were acquired with an EMCCD camera (Andor Technology Ltd, UK) and processed with ZEN software, exposure times varied between 100 and $120 \mathrm{~ms}$. Three-dimensional images were generated using a z-step of $150 \mathrm{~nm}$ (total thickness $\sim 5 \mu \mathrm{m}$ ), while reconstructions and co-distributions were determined with IMARIS software (Bitplane). For co-distribution analysis, we used the colocalization module implemented in the IMARIS software. To process the images, we first applied a threshold for each channel (threshold adapted according to the labelling). For the Alexa488 channel, we used a threshold fixed at 15,000. For the red channel, the threshold varied between 8,000 and 25,000 , depending on the dye used. The percentage (\%) of volume B (green channel) above the threshold that colocalized (colocalized volume B above threshold/total volume B above threshold) and thresholded Mander's coefficient B and Pearson coefficient were calculated. The acquisition was performed sequentially using 43HE, 38HE and BP 420-480 Zeiss filter sets, and 15 frames were acquired to reconstruct one image (five rotations $\times$ three phases, with a SIM grating period of $51 \mu \mathrm{m}$ for the blue channel, $42 \mu \mathrm{m}$ for the green channel and $34 \mu \mathrm{m}$ for the red channel). Beads with a diameter of $100 \mathrm{~nm}$ were imaged to measure the chromatic misalignment of our system (fit procedure by the ZEN software), and these parameters enabled further correction of the alignment on each acquired multi-channel stack. Image reconstruction was achieved using ZEN software with the following parameters: Noise Level -4, Sectioning 99-83-83 and Frequency Weighting 1.

Specifically, iKoTgVps35 mutants transiently transfected were treated with ATc or left untreated for $24 \mathrm{~h}$ or $48 \mathrm{~h}$, fixed and stained with primary specific antibodies and either Dylight594- or Alexa488-conjugated secondary antibodies. Forty eight hours of ATc treatment corresponds to the time point where optimal effects on rhoptry and microneme biogenesis were observed and expression of $\mathrm{TgVps} 35$ protein was completely ablated as demonstrated by western blots and IFA. Therefore, all other experiments were then performed at this time point in this study. For GRASP-RFP expressing parasites, the fluorescence signal was directly visualized by SIM. Colocalization was quantified as overlap between green and red images using the IMARIS software for 90 intracellular parasites from 7 to 8 vacuoles.

Electron microscopy. For transmission electron microscopy, intracellular tachyzoites of $\mathrm{iKoTgVps} 35$, complemented mutants, or parental parasites were fixed overnight at $4{ }^{\circ} \mathrm{C}$ with $2.5 \%$ glutaraldehyde $/ 4 \%$ paraformaldehyde prepared $0.1 \mathrm{M}$ cacodylate buffer, cells were fixed in $2.5 \%$ glutaraldehyde prepared in $0.1 \mathrm{M}$ cacodylate buffer and post-fixed in $1 \%$ osmium tetroxide in the same buffer. After acetonitrile dehydration, the pellet was embedded in Epon. Ultrathin sections $(90 \mathrm{~nm})$ were cut using a Leica UC7 ultramicrotome and collected on 150 mesh hexagonal barred copper grids. After staining with $2 \%$ uranyl acetate prepared in $50 \%$ ethanol and incubation with a lead citrate solution, sections were observed on a Hitachi H-600 transmission electron microscope at $75 \mathrm{kV}$ accelerating voltage.

Host cell invasion assays. The conditional iKoTgVps35 mutants were treated with ATc or left untreated for $48 \mathrm{~h}$ and then used to infect HFF cells for $1 \mathrm{~h}$ at $37^{\circ} \mathrm{C}$. Twenty-four hours post-infection, coverslips were fixed and processed for immunofluorescence assays. The intracellular parasites were labelled with antiSAG1 antibodies, and the nucleus was stained with 4', 6-diamidino-2-phenylindole. For each condition, 400 microscopic fields $(\times 40)$ were observed. The number of SAG1-positive parasites was compared with the number of cells stained with $4^{\prime}$, 6-diamidino-2-phenylindole. The ratio parasites/cells are presented as mean values \pm s.d. from three independent experiments.

Mouse infectivity studies. A group of 4 female BALB/C mice (6-8-week-old, from Janvier Labs, France) were intraperitoneally injected with $2 \times 10^{6}$ tachyzoites of RH TaTi, iKo $T g V p s 35$ or Comp-iKo $T g V p s 35$ parasites. To suppress $T g V p s 35$ expression, the drinking water was supplemented with $0.2 \mathrm{mg} \mathrm{ml}^{-1}$ of ATc and $5 \%$ of sucrose. As controls, a group of four mice was also infected with the same parasite lines without ATc. Survival was monitored over 4 weeks. For vaccination assays, BALB/C mice were infected by intraperitoneal injection with $3 \times 10^{2}$ tachyzoites of $\mathrm{RH} \mathrm{TaTi}$, iKoTgVps35 or Comp-iKoTgVps35 parasites followed by ATc treatment or not. After 4 weeks, the survived mice were re-challenged with $1 \times 10^{3}$ tachyzoites of wild-type RH strain and survival monitored for 30 days. All animal experiments were performed following the guidelines of the Pasteur Institute of Lille animal study board, which conforms to the Amsterdam Protocol on animal protection and welfare, and Directive 86/609/EEC on the Protection of Animals Used for Experimental and Other Scientific Purposes, updated in the Council of Europe's Appendix A (http://conventions.coe.int/Treaty/EN/Treaties/ PDF/123-Arev.pdf). The animal work also complied with the French law (no. 87-848 dated 19 october 1987) and the European Communities Amendment of Cruelty to Animals Act 1976. All animals were fed with regular diet and all procedures were in accordance with national regulations on animal experimentation and welfare authorized by the French Ministry of Agriculture and Veterinary committee (permit number: 59-009145).

Co-immunoprecipitation and western blots. Tachyzoites $\left(1 \times 10^{9}\right.$ parasites $)$ from $T g V p s 35-H A, T g V p s 26-H A, T g V p s 29-c M y c$ and $\mathrm{RH} \Delta$ ku80 strains were lysed with $10 \mathrm{mM}$ HEPES, pH 7.9; $1.5 \mathrm{mM} \mathrm{MgCl} ; 10 \mathrm{mM} \mathrm{KCl} ; 0.5 \mathrm{mM}$ dithiothreitol (DTT); $0.1 \mathrm{mM}$ EDTA; 0.65\% NP40; $0.5 \mathrm{mM}$ phenylmethanesulfonylfluoride (PMSF); and a cocktail of protease inhibitors (Sigma Aldrich). After $30 \mathrm{~min}$ on ice, the lysates were centrifuged at $21,693 \mathrm{~g}$ for $30 \mathrm{~min}$ at $4{ }^{\circ} \mathrm{C}$, and the supernatants were then incubated with anti-HA or anti-cMyc agarose beads (Thermo Pierce) overnight at $4{ }^{\circ} \mathrm{C}$ under rotating shaker. After five washings with $10 \mathrm{mM}$ Tris, $\mathrm{pH} 7.5 ; 150 \mathrm{mM} \mathrm{NaCl} ; 0.2 \%$ Triton X-100; $0.5 \mathrm{mM}$ PMSF, and a final washing with $62.5 \mathrm{mM}$ Tris, $\mathrm{pH} 6.8$, immunoprecipitates were eluted with Laemmli buffer (0.2\% SDS, $100 \mathrm{mM}$ DTT, and $10 \%$ sucrose). These samples were analysed by SDS-polyacrylamide gel electrophoresis (SDS-PAGE) followed by silver staining before proteomics analyses. For western blots, parasites were lysed with Laemmli 
buffer, and lysate proteins were separated on $12 \%$ acrylamide gels and processed for immunoblotting using primary specific antibodies and then secondary antibodies conjugated to alkaline phosphatase (Thermo Pierce). All primary antibodies used for western blots were listed in Supplementary Table 5. The blots were imaged using ChemiDoc XRS ${ }^{+}$(Bio-Rad).

Liquid chromatography coupled to mass spectrometry. For liquid chromatography and mass spectrometry performed using Q-Exactive mass spectrometer (Thermo Scientific, Bremen, Germany), each sample (30 $\mu$ l) was denatured with Laemmli sample buffer and loaded onto one-dimensional SDS-PAGE (12\%), stained with colloidal Coomassie G-250 (Bio-Rad, Hercules, CA) and six slices per sample were excised for reduction and alkylation. For this step, gel slices were cut in small pieces $\left(1 \mathrm{~mm}^{3}\right)$ and the staining of gel pieces were removed thrice with $120 \mu \mathrm{l}$ of a mixture of 50/50 (v/v), $25 \mathrm{mM}$ ammonium bicarbonate $\left(\mathrm{NH}_{5} \mathrm{CO}_{3}\right) /$ acetonitrile for $10 \mathrm{~min}$. In-gel reduction and alkylation of protein disulfide bonds were performed, respectively, with $100 \mu \mathrm{l}$ of $10 \mathrm{mM}$ of DTT in $50 \mathrm{~min}$ at $57^{\circ} \mathrm{C}$ and $100 \mu \mathrm{l}$ of $50 \mathrm{mM}$ of iodoacetamide (IAM) for $30 \mathrm{~min}$ at room temperature. After a washing step with $120 \mu \mathrm{l}$ of $25 \mathrm{mM} \mathrm{NH}_{5} \mathrm{CO}_{3}$ and the dehydration step with $100 \mu \mathrm{l}$ acetonitrile for $5 \mathrm{~min}$, an in-gel digestion was performed on each sample with $0.07 \mu \mathrm{g}$ of sequencing grade porcine trypsin (Promega, Madison, WI) for $16 \mathrm{~h}$ at $37^{\circ} \mathrm{C}$. The peptide were extracted thrice from gel with a mixture of $60 / 40 / 0.1$ $(\mathrm{v} / \mathrm{v} / \mathrm{v})$, acetonitrile $/ 25 \mathrm{mM}$ of $\mathrm{NH}_{5} \mathrm{CO}_{3}(\mathrm{v} / \mathrm{v})$ and $0.1 \%$ formic acid. The extracted solution were then dried with vacuum centrifuge (Uniequip $\mathrm{GmbH}$, Munich, Germany) and resuspended in $10 \mu \mathrm{l}$ of water containing $0.1 \%$ formic acid

Each extracted solution of $3 \mu \mathrm{l}$ was injected into the Ultimate 3,000 RSLC nano- System (Dionex, Thermo Scientific) through a trap column (Acclaim PepMap, $5 \mathrm{~mm} \times 300 \mu \mathrm{m}$ inner diameter, C18, $5 \mu \mathrm{m}, 100 \AA$; Dionex) at $5 \mu \mathrm{min}^{-1}$ with water containing $0.1 \%$ formic acid and $2 \%$ acetonitrile. After $5 \mathrm{~min}$, the trap column was set on-line with analytical column (Acclaim PepMap RSLC, $15 \mathrm{~cm} \times 75 \mu \mathrm{m}$ inner diameter, C18, $2 \mu \mathrm{m}, 100 \AA \AA$; Dionex, Sunnyvale, CA). The elution was carried out by applying mixture of solvent A (HPLC grade water with $0.1 \%$ formic acid) and solvent B (HPLC grade acetonitrile with $0.1 \%$ formic acid) at the flow rate of $300 \mathrm{nl} \mathrm{min}-1$. The separations were performed by applying a linear gradient of $2-40 \%$ solvent B over 38 min followed by a washing step (5 min at $70 \%$ solvent B) and an equilibration step (11 min at $2 \%$ solvent B).

The eluted peptides were analysed by a Q-Exactive mass spectrometer. For ionization, a nanospray Flex Ion Source was used with a voltage of $1.9 \mathrm{kV}$, and the capillary temperature was $275^{\circ} \mathrm{C}$. Full MS scans were acquired in the Orbitrap mass analyser over $\mathrm{m} / \mathrm{z} 300-3,500$ range with resolution of 70,000 at $\mathrm{m} / \mathrm{z} 200$. The target automatic gain control value of $1 \times 10^{6}$ was used with a maximum allowed injection time (Maximum IT) of $250 \mathrm{~ms}$. For MS/MS, an isolation window of $2 \mathrm{~m} / \mathrm{z}$ was utilized. Ten most intense peaks (TopN) with charge state between 2 and 6 were selected for fragmentation in the higher-energy collisional induced dissociation cell with normalized collision energy of 35 . The tandem mass spectra were acquired over $m / z$ 200-2,000 range in the Orbitrap mass analyser with resolution 35,000 at $\mathrm{m} / \mathrm{z} 200$ and an automatic gain control of $2 \times 10^{5}$. The ion intensity selection threshold was $6.7 \times 10^{4}$, and the maximum injection time was $150 \mathrm{~ms}$. The dynamic exclusion time was $10 \mathrm{~s}$ and. the total run time was $60 \mathrm{~min}$. All these systems were fully controlled by Thermo Xcalibur 3.0 (Thermo Fisher Scientific).

All data files ( ${ }^{*}$.raw) collected were processed with a specific workflow designed in Proteome Discoverer 1.4 (Thermo Fisher Scientific). MS/MS data was interpreted using two search engine Mascot (version 2.4.1, Matrix Science, London, UK) and Sequest HT (Thermo Fisher Scientific). Searches were performed against T. gondii (TGVEG, TGME49 and TGGT1 stain) protein sequences downloaded from www.toxodb.org at the 11th December 2014 (18,954 entries). The Mascot ion score were $>20$ and Sequest HT XCorr $>1.5$. The target-decoy database search allowed us to control and to estimate the false positive identification rate ${ }^{51}$.

\section{Nanoscale liquid chromatography and tandem mass spectrometry.}

For nanoscale liquid chromatography coupled to tandem mass spectrometry (NanoLC-MS/MS), samples were electrophoresed onto $12 \%$ SDS-PAGE and stained overnight with colloidal Coomassie Brilliant Blue. Gel bands were manually excised, reduced $1 \mathrm{~h}$ at $60^{\circ} \mathrm{C}$ by adding DTT to a final concentration of $10 \mathrm{mM}$ and alkylated by adding iodoacetamide to a final concentration of $40 \mathrm{mM}$. An overnight digestion was performed by adding trypsin (Promega). Tryptic peptides were extracted $(60 \%$ acetonitrile, $0.1 \% \mathrm{HCOOH})$ before mass spectrometry analyses. NanoLC-MS/MS analyses were performed on three different systems: nanoACQUITY Ultra-Performance-LC system (UPLC; Waters, Milford, MA, USA) hyphenated to either Q-TOF Impact HD or MaXis 4G (Bruker Daltonics, Bremen, Germany) and a nanoLC-Chip/Cube (Agilent Technologies, Palo Alto, CA, USA) hyphenated to an ion trap amaZon (Bruker Daltonics). For maXis 4G and amaZon analysis, methods used were previously described ${ }^{52}$ with slight modifications. For Impact HD analysis, peptides were first trapped on a $0.18 \times 20 \mathrm{~mm}^{2}, 5 \mu \mathrm{m}$ Symmetry C18 pre-column (Waters), and then separated on an ACQUITY UPLC BEH130 C18 column (Waters), $75 \mu \mathrm{m} \times 250 \mathrm{~mm}$ with $1.7 \mu \mathrm{m}$ particle size. The solvent system consisted of $0.1 \% \mathrm{HCOOH}$ in water (solvent $\mathrm{A}$ ) and $0.1 \% \mathrm{HCOOH}$ in acetonitrile (solvent B). Trapping was performed for $3 \mathrm{~min}$ at $5 \mu \mathrm{lmin}^{-1}$ with $99 \% \mathrm{~A}$ and $1 \% \mathrm{~B}$. Elution was performed at a flow rate of $450 \mathrm{nl} \mathrm{min}^{-1}$, using a $1-35 \%$ gradient (solvent B) over $30 \mathrm{~min}$ at $60^{\circ} \mathrm{C}$.
The mass spectrometer was equipped with a Captive Spray source and a nano-Booster operating in positive mode, with the following settings: source temperature was set at $150^{\circ} \mathrm{C}$ while drying gas flow was at $31 \mathrm{~min}^{-1}$. The nano-electrospray voltage was optimized to $-1,300 \mathrm{~V}$. External mass calibration of the time-of-flight (TOF) was achieved before each set of analyses using Tuning Mix (Agilent Technologies) in the mass range of $322-2,722 \mathrm{~m} / z$. Mass correction was achieved by recalibration of acquired spectra to the applied lock masses hexakis $\left(2,2,3,3\right.$,-tetrafluoropropoxy) phosphazine $\left.\left([\mathrm{M}+\mathrm{H}]^{+}=922.0098 \mathrm{~m} / z\right)\right]$.

For tandem MS experiments (CID), the system was operated with fixed cycle time of $3 \mathrm{~s}$ in the range of $150-2,200 \mathrm{~m} / \mathrm{z}$. MS/MS scan speed was monitored in function of precursor intensity from 4 to $25 \mathrm{~Hz}$. Ions were excluded after acquisition of one MS/MS spectra and the exclusion was released after $1 \mathrm{~min}$. The complete system was fully controlled by Hystar 3.2 (Bruker Daltonics).

\section{Bioinformatics and protein identification. Mass data collected during} nanoLC-MS/MS analyses were processed, converted into '.mgf files with Data Analysis 4.0 (Bruker Daltonics) and interpreted using MASCOT 2.5.1 algorithm (Matrix Science, London, UK) running on a local server. Searches were performed without any molecular weight or isoelectric point restrictions against an in-house generated protein database composed of protein sequences of T. gondii (ToxoDB database, October 2014) and known contaminant proteins such as human keratins and trypsin. All proteins were concatenated with reversed copies of all sequences (49,328 entries) with an in-house database generation toolbox https://msda.unistra.fr ${ }^{53}$. Trypsin was selected as enzyme, carbamidomethylation of cysteine $(+57 \mathrm{Da})$ was set as fixed modification, oxidation of methionine $(+16 \mathrm{Da})$ were set as variable modification and both precursor and fragment mass tolerances were adapted according to instrumental mass accuracy. Mascot results were loaded into the Proline software (Proline Studio Release 1.0) and filtered to obtain a false discovery rate of $<1 \%$.

Absolute quantitation using LC-SRM. For microLC-SRM assay, three proteotypic peptides per targeted protein ( $T g V p s 35, T g V p s 26$ and $T g V p s 29)$ were selected. A total of nine high-purity isotopically labelled equivalent peptides were purchased (AQUA peptides, Thermo Fischer Scientific, Bremen; Germany). Previous nanoLC-MS/MS analyses afforded a representative MS/MS spectrum for each peptide. Four to six transitions were monitored for both endogenous and heavy-labelled peptides. Thus, a total of 78 transitions corresponding to 20 precursors and 3 proteins were measured. For the lower limits of quantification and detection determination, a dilution series of the labelled peptides was realized at different concentrations in a mixture containing all tagged proteins and injected in triplicate on a QQQ-6490 triple quadrupole mass spectrometer (Agilent Technologies). The area under curve of the three best transitions per peptide were summed and drawn versus the peptide concentration. Two calibration curves were drawn per peptide: high calibration curve $\left(15 \mathrm{fmol} \mu \mathrm{l}^{-1}-238 \mathrm{fmol} \mu \mathrm{l}^{-1}\right)$ and low-calibration curve $\left(2 \mathrm{fmol} \mu \mathrm{l}^{-1}-30 \mathrm{fmol} \mu \mathrm{l}^{-1}\right)$. We evaluated the lower limits of quantification and the lower limits of detection determination by applying recognized definitions $s^{54}$.

For the SRM analyses, samples were electrophoresed onto 4\% SDS-PAGE and stained for $45 \mathrm{~min}$ with colloidal Coomassie Brilliant Blue. The stacking gel bands were predigested and digested as previously described and $1 \mu \mathrm{l}$ of a mixture of heavy-labelled peptides was added to each sample before LC-SRM analyses.

All separations were carried out on an Agilent 1100 Series HPLC system (Agilent Technologies). For each analysis, the sample was loaded into a trapping column ZORBAX 300SB-C18 MicroBore Guard $5 \mu \mathrm{m}, 1.0 \times 17 \mathrm{~mm}^{2}$ (Agilent Technologies) at $50 \mu \mathrm{lmin}^{-1}$ with aqueous solution containing $0.1 \%(\mathrm{v} / \mathrm{v})$ formic acid and $2 \%$ acetonitrile. After 3 min trapping, the column was put on-line with a ZORBAX 300SB-C18 $3.5 \mu \mathrm{m}, 0.3 \times 150 \mathrm{~mm}^{2}$ column (Agilent Technologies). Peptide elution was performed at $5 \mu \mathrm{lmin}{ }^{-1}$ by applying a linear gradient of solvent A (water with $2 \%$ acetonitrile and $0.1 \%(\mathrm{v} / \mathrm{v})$ formic acid) and B (acetonitrile with $0.1 \%$ (v/v) formic acid), from 8 to $42 \%$ solvent B over $30 \mathrm{~min}$ followed by a washing step ( 2 min at $90 \%$ solvent B) and an equilibration step (13 min at $8 \%$ solvent B). The isolation width for both Q1 and Q3 was set to $0.7 \mathrm{~m} / z$ unit. The collision energy was experimentally optimized by testing nine values centred on the calculated value from the one given by the supplier. Time-scheduled SRM method targeted the pairs of isotopically labelled peptides/ endogenous peptides in $\pm 5 \mathrm{~min}$ retention time windows within a cycle time of $3 \mathrm{~s}$. Mass data collected during LC-SRM were processed with the Skyline open-source software package 3.1.1 (ref. 55). Area intensity ratios of the heavy and the light forms of each peptide were manually checked. The endogenous peptide amount calculation was performed by using the most suitable calibration curve. The mass spectrometry and LC-SRM data were deposited to the ProteomeXchange ${ }^{56}$ Consortium via PRIDE ${ }^{57}$ partner repository, and the peptide atlas SRM experiment library (PASSEL), respectively.

Production of recombinant glutathione S-transferase (GST)-TgHP12 and specific antibodies. The DNA corresponding to C-terminal sequence of 254 amino acids long from 1,051 to 1,812 nucleic acid was amplified by PCR using the following primers: sense $5^{\prime}$-CCGGGGATCCGTAGAAAAGCCTACAACGGTG GGG-3', and antisense, 5'-CCGGGCGGCCGCTCACAATCTGTCAAGTCTTCCT 
CCAGTC- ${ }^{\prime}$. The amplified DNA was purified and cloned in frame into pGEX6P3. After verification by sequencing, the plasmid was used to transform E. coli BL21 for recombinant protein expression. Protein was purified by GST column and $100 \mu \mathrm{g}$ of protein was used to immunize one Wistar (RjHan:WI) rat (Janvier Labs, France) using complete Freund adjuvant. The rat was challenged three times with $50 \mu \mathrm{g}$ of protein and incomplete Freund adjuvant before bleeding 10 days after the last boost and the serum was purified.

GST-pull down experiments. Recombinant Rab5B, Rab7 and Rab11B proteins were fused to GST using pGEX6P3. After transformation of BL21 E. coli, lysates were incubated to Glutathione-beads and washed four times with buffer A: $50 \mathrm{mM}$ Tris-HCl pH 7.5, 0.5M NaCl, $270 \mathrm{mM}$ sucrose $1 \mathrm{mM}$ EGTA, $1 \mathrm{mM}$ EDTA, $1 \%$ Triton X-100 and $0.5 \mathrm{mM}$ PMSF) and washed six time with buffer A without Triton X-100. Total $T g$ Vps35-HA and $T g V p s 26-H A$ knock-in parasite extracts were prepared from $10^{9}$ tachyzoites that were lysed with buffer B: $10 \mathrm{mM}$ HEPES $\mathrm{pH} 7.91 .5 \mathrm{mM} \mathrm{MgCl}_{2}, 10 \mathrm{mM} \mathrm{KCl}, 0.5 \mathrm{mM}$ DTT, $0.1 \mathrm{mM}$ EDTA, $0.65 \% \mathrm{NP} 40$ and $0.5 \mathrm{mM}$ PMSF. The parasite lysate (equivalent to $2.0 \times 10^{8}$ tachyzoites) were added in pull down buffer containing $50 \mathrm{mM}$ Tris- $\mathrm{HCl} \mathrm{pH} \mathrm{7.5,150} \mathrm{mM} \mathrm{NaCl}$ and $0.5 \mathrm{mM}$ PMSF and incubated with beads containing $2 \mu \mathrm{g}$ GST-Rab5, GST-Rab7, GST-Rab11B or GST alone in the presence of $1 \mathrm{mM}$ GTP or GDP overnight at $4{ }^{\circ} \mathrm{C}$. Precipitants were washed three time with the pull down buffer containing $0.1 \%$ Triton X-100 and eluted by Laemmli buffer and analysed by western blots, which were probed with anti-HA antibodies and anti-GST.

Statistical analysis. All data were analysed with Graph Pad Prism software. A Student's $t$-test was used for statistical analysis. The Mann-Whitney test was also used for analysis of mice survival curves.

\section{References}

1. Pappas, G., Roussos, N. \& Falagas, M. E. Toxoplasmosis snapshots: Global status of Toxoplasma gondii seroprevalence and implications for pregnancy and congenital toxoplasmosis. Int. J. Parasitol. 39, 1385-1394 (2009).

2. Hager, K. M., Striepen, B., Tilney, L. G. \& Roos, D. S. The nuclear envelope serves as an intermediary between the ER and Golgi complex in the intracellular parasite Toxoplasma gondii. J. Cell Sci. 112, 2631-2638 (1999).

3. Tomavo, S., Slomianny, C., Meissner, M. \& Carruthers, V. B. Protein trafficking through the endosomal system prepares intracellular parasites for a home invasion. PLoS Pathog. 9, e1003629 (2013).

4. Breinich, M. S. et al. A dynamin is required for the biogenesis of secretory organelles in Toxoplasma gondii. Curr. Biol. 19, 277-286 (2009).

5. Kremer, K. et al. An overexpression screen of Toxoplasma gondii Rab-GTPases reveals distinct transport routes to the micronemes. PLoS Pathog. 9, e1003213 (2013).

6. Jackson, A. J., Clucas, C., Mamczur, N. J., Ferguson, D. J. \& Meissner, M. Toxoplasma gondii syntaxin 6 is required for vesicular transport between endosomal-like compartments and the Golgi complex. Traffic 14, 1166-1181 (2013).

7. Morion-Guyot, J., Pastore, S., Berry, L., Lebrun, M. \& Daher, W. Toxoplasma gondii Vps11, a subunit of HOPS and CORVET tethering complexes is essential for the biogenesis of secretory organelles. Cell. Microbiol. 17, 1157-1178 (2015).

8. Mogelsvang, S., Marsh, B. J., Ladinsky, M. S. \& Howell, K. E. Predicting function from structure: 3D structure studies of the mammalian Golgi complex. Traffic 5, 338-345 (2004).

9. He, C. Y. Golgi biogenesis in simple eukaryotes. Cell. Microbiol. 9, 566-572 (2007).

10. Brydges, S. D., Harper, J. M., Parussini, F., Coppens, I. \& Carruthers, V. B. A transient forward-targeting element for microneme-regulated secretion in Toxoplasma gondii. Biol. Cell 100, 253-264 (2008).

11. Parussini, F., Coppens, I., Shah, P. P., Diamond, S. L. \& Carruthers, V. B. Cathepsin L occupies a vacuolar compartment and is a protein maturase within the endo/exocytic system of Toxoplasma gondii. Mol. Microbiol. 76, 1340-1357 (2010).

12. Dubremetz, J.-F. Rhoptries are major players in Toxoplasma gondii invasion and host cell interaction. Cell Microbiol. 9, 841-848 (2007).

13. Schmidt, O. \& Teiss, D. The ESCRT machinery. Curr. Biol. 22, R116-R120 (2012).

14. Lige, B. et al. Introduction of caveolae structural proteins into the protozoan Toxoplasma results in the formation of heterologous caveolae but not caveolar endocytosis. PLoS ONE 12, e51773 (2012).

15. Pieperhoff, M. S., Schmitt, M., Ferguson, D. J. \& Meissner, M. The role of clathrin in post-Golgi trafficking in Toxoplasma gondii. PLoS ONE 8, e77620 (2013).

16. Dou, Z., McGovern, O. L., Di Cristina, M. \& Carruthers, V. B. Toxoplasma gondii ingests and digests host cytosolic proteins. MBio 5, e01188-14 (2014).

17. Sloves, P. J. et al. Toxoplasma sortilin-like receptor regulates protein transport and is essential for apical secretory organelle biogenesis and host infection. Cell Host Microbe 11, 515-527 (2012).

18. Bonifacino, J. S. \& Hurley, J. H. Retromer. Curr. Opin. Cell Biol. 20, 427-436 (2008).
19. Gallon, M. \& Cullen, P. J. Retromer and sorting nexins in endosomal sorting. Biochem. Soc. Trans. 43, 33-47 (2015).

20. Norwood, S. J. et al. Assembly and solution structure of the core retromer protein complex. Traffic 12, 56-71 (2011).

21. Agop-Nersesian, C. et al. Biogenesis of the inner membrane complex is dependent on vesicular transport by the alveolate specific GTPase Rab11B. PLoS Pathog. 6, e1001029 (2010).

22. Seaman, M. N. J., Harbour, M. E., Tattersall, D., Read, E. \& Bright, N. Membrane recruitment of the cargo-selective retromer subcomplex is catalyzed by the small GTPase Rab7 and inhibited by the Rab-GAP TBC1D5. J. Cell Sci. 122, 2371-2382 (2009)

23. Fox, B. A. \& Bzik, D. J. De novo pyrimidine biosynthesis is required for virulence of Toxoplasma gondii. Nature 415, 926-929 (2002).

24. Tomavo, S., Schwarz, R. T. \& Dubremetz, J.-F. Evidence for glycosylphosphatidylinositol anchoring of Toxoplasma gondii major surface antigens. Mol. Cell. Biol. 9, 4576-4580 (1989).

25. Di Crisanti, M., Spaccapelo, R., Soldati, D., Bistoni, F. \& Crisanti, A. Two conserved amino acid motifs mediate protein targeting to the micronemes of the apicomplexan parasite Toxoplasma gondii. Mol. Cell. Biol. 20, 7332-7341 (2000)

26. Arighi, C. N. et al. Role of the mammalian retromer in sorting of the cationindependent mannose 6-phosphate receptor. J. Cell Biol. 165, 123-133 (2004).

27. Seaman, M. N. J. The retromer complex-endosomal protein recycling and beyond. J. Cell Sci. 125, 4693-4702 (2012).

28. Steinberg, F. et al. A global analysis of SNX27-retromer assembly and cargo specificity reveals a function in glucose and metal ion transport. Nat. Cell Biol. 15, 461-471 (2013).

29. Maruzs, T. et al. Retromer ensures the degradation of autophagic cargo by maintaining lysosome function in Drosophila. Traffic 16, 1088-1107 (2015).

30. Eathiraj, S., Pan, X., Ritacco, C. \& Lambright, D. G. Structural basis of familywide Rab GTPase recognition by rabenosyn-5. Nature 436, 415-419 (2005).

31. Lall, P. et al. Structural and functional analysis of FIP2 binding to the endosome-localised Rab25 GTPase. Biochim. Biophys. Acta 1834, 2679-2690 (2013).

32. Kelley, L. A., Mezulis, S., Yates, C. M., Wass, M. N. \& Sternberg, M. J. The Phyre2 web portal for protein modeling, prediction and analysis. Nat. Protoc. 10, 845-858 (2015).

33. Law, C. J., Maloney, P. C. \& Wang, D. N. Ins and outs of major facilitator superfamily antiporters. Annu. Rev. Microbiol. 62, 289-305 (2008).

34. McGough, I. J. et al. Identification of molecular heterogeneity in SNX27-retromer-mediated endosome-to-plasma membrane recycling. J. Cell Sci. 127, 4940-4953 (2014).

35. Cullen, P. J. Endosomal sorting and signalling: an emerging role for sorting nexins. Nat. Rev. Mol. Cell Biol. 9, 574-582 (2008).

36. Harrison, M. S. et al. A mechanism for retromer endosomal coat complex assembly with cargo. Proc. Natl Acad. Sci. USA 111, 267-272 (2014).

37. Rojas, R. et al. Regulation of retromer recruitment to endosomes by sequential action of Rab5 and Rab7. J. Cell Biol. 183, 513-526 (2008).

38. Liu, T. T., Gomez, T. S., Sackey, B. K., Billadeau, D. D. \& Burd, C. G. Rab GTPase regulation of retromer-mediated cargo export during endosome maturation. Mol. Biol. Cell. 23, 2505-2515 (2013).

39. Swarbrick, J. D. et al. VPS29 is not an active metallo-phosphatase but is a rigid scaffold required for retromer interaction with accessory proteins. PLoS ONE 6, e20420 (2011).

40. Nakada-Tsukui, K., Saito-Nakano, Y., Ali, V. \& Nozaki, T. A retromer-like complex is a novel Rab7 effector that is involved in the transport of the virulence factor cysteine protease in the enteric protozoan parasite Entamoeba histolytica. Mol. Biol. Cell 16, 5294-5303 (2005).

41. Pao, S. S., Paulsen, I. T. \& Saier, M. H. Major facilitator superfamily. Microbiol. Mol. Biol. Rev. 62, 1-34 (1998).

42. Cullen, P. J. \& Korswagen, H. C. Sorting nexins provide diversity for retromer-dependent trafficking events. Nat. Cell Biol. 14, 29-37 (2011).

43. Gautreau, A., Oguievetskaia, K. \& Ungermann, C. Function and regulation of the endosomal fusion and fission machineries. Cold Spring Harb. Perspect. Biol. 1, 6 (2014).

44. Miranda, K. et al. Characterization of a novel organelle in Toxoplasma gondii with similar composition and function to the plant vacuole. Mol. Microbiol. 76, 1358-1375 (2010).

45. Tomavo, S. Evolutionary repurposing of endosomal systems for apical organelle biogenesis in Toxoplasma gondii. Int. J. Parasitol. 44, 133-138 (2014).

46. Huynh, M. H. \& Carruthers, V. B. Tagging of endogenous genes in a Toxoplasma gondii strain lacking Ku80. Eukaryot. Cell 8, 530-539 (2009).

47. Sheiner, L. et al. A systematic screen to discover and analyze apicoplast proteins identifies a conserved and essential protein import factor. PLoS Pathog. 7, e1002392 (2011).

48. Cong, L. et al. Multiplex genome engineering using CRISPR/Cas systems. Science 339, 819-823 (2013).

49. Weber, E. et al. Assembly of designer TAL effectors by Golden Gate cloning. PloS ONE 6, e19722 (2011) 
50. Sloves, P. J. et al. Apical organelle secretion by Toxoplasma controls innate and adaptive immunity and mediates long-term protection. J. Infect. Dis. 212, 1449-1458 (2015)

51. Elias, J. E. \& Gygi, S. P. Target-decoy search strategy for increased confidence in large-scale protein identifications by mass spectrometry. Nat. Methods $\mathbf{4}$, 207-214 (2007).

52. Gissot, M. et al. Toxoplasma gondii Alba proteins are involved in translational control of gene expression. J. Mol. Biol. 425, 1287-1301 (2013).

53. Carapito, C. et al. MSDA, a proteomics software suite for in-depth Mass Spectrometry Data Analysis using grid computing. Proteomics 14, 1014-1019 (2014).

54. Domanski, D. et al. MRM-based multiplexed quantitation of 67 putative cardiovascular disease biomarkers in human plasma. Proteomics 12, 1222-1243 (2012).

55. MacLean, B. et al. Skyline: an open source document editor for creating and analyzing targeted proteomics experiments. Bioinformatics 26, 966-968 (2010).

56. Vizcaíno, J. A. et al. ProteomeXchange provides globally co-ordinated proteomics data submission and dissemination. Nat. Biotechnol. 30, 223-226 (2014).

57. Vizcaíno, J. A. et al. 2016 update of the PRIDE database and related tools. Nucleic Acids Res. 44, 447-456 (2016).

\section{Acknowledgements}

L.O.S. would like to thank Thomas Mouveaux, Pasteur Institute of Lille; Etienne Dewailly, University of Lille; Joelle Duflot, Pasteur Institute of Lille for excellent technical assistance and Pierre Julien Sloves, Pasteur Institute of Lille for his early guidance. This work was supported by the following grants: Laboratoire d'Excellence (LabEx) ParaFrap from the National Agency for Research ANR-11-LABX-0024 and the ANR-14-CE140002-01. We also acknowledge additional financial support from the INSERM, Pasteur Institute of Lille and CNRS. PhD and Post-doc fellowships were from 'Métropole Européenne de Lille' (MEL) to L.O.S. and 'FEDER-Région' for T.D.A. M.A.H is supported by the European Research Council (ERC Consolidator grant no. 614880).

\section{Author contributions}

L.S.O., T.D.A., B.W., A.H., I.C., C.S.R. and A.V.D. conceived and designed the experiments, analysed data and wrote paper; F.S. and M.A.H.: engineering CRISPR-Cas9 vectors; E.W. and F.L.: SIM imaging; C.S.: electron microscopy; S.T. designed, supervised and wrote this study.

\section{Additional information}

Accession codes: The mass spectrometry proteomics data collected during nanoLC-MS/MS analyses have been deposited to the ProteomeXchange Consortium via PRIDE partner repository with the data set identifier PXD003603. The LC-SRM data have been deposited in the Peptide Atlas SRM Experiment Library (PASSEL) with the data set identifier PASS00824.

Supplementary Information accompanies this paper at http://www.nature.com/ naturecommunications

Competing financial interests: The authors declare no competing financial interest.

Reprints and permission information is available online at http://npg.nature.com/ reprintsandpermissions/

How to cite this article: Sangaré, L. O. et al. Unconventional endosome-like compartment and retromer complex in Toxoplasma gondii govern parasite integrity and host infection. Nat. Commun. 7:11191 doi: 10.1038/ncomms11191 (2016).

\section{(c) (1)}

This work is licensed under a Creative Commons Attribution 4.0 International License. The images or other third party material in this article are included in the article's Creative Commons license, unless indicated otherwise in the credit line; if the material is not included under the Creative Commons license, users will need to obtain permission from the license holder to reproduce the material To view a copy of this license, visit http://creativecommons.org/licenses/by/4.0/ 